\title{
Trapping of neutral molecules by the beam electromagnetic field
}

\author{
G. Franchetti®* \\ GSI Helmholtzzentrum für Schwerionenforschung GmbH, 64291 Darmstadt, Germany, \\ Goethe University Frankfurt, Max-von-Laue-Straße 7, 60438 Frankfurt am Main, Germany, \\ and Helmholtz Research Academy Hesse for FAIR (HFHF), GSI Helmholtz Center, \\ Campus Frankfurt, Max-von-Laue-Straße 12, 60438 Frankfurt, Germany \\ F. Zimmermann 1 \\ European Organization for Nuclear Research (CERN), \\ Esplanade des Particules 1, 1217 Meyrin, Switzerland \\ M. A. Rehman $\odot$ \\ High Energy Accelerator Research Organization (KEK), \\ 1-1 Oho, Tsukuba, Ibaraki, Japan, 305-0801, Japan, \\ and The Graduate University for Advanced Studies (SOKENDAI), \\ School of High Energy Accelerator Science, Tsukuba, Ibaraki 305-0801, Japan
}

(Received 12 January 2021; accepted 8 March 2021; published 12 May 2021)

\begin{abstract}
Neutral uncharged molecules are affected by the electromagnetic field of a charged particle beam if they carry either an electric or a magnetic dipole moment. The residual gas in an accelerator beam pipe consists of such molecules. In this paper we study their dynamics. Under a few approximations, whose validity we explore and justify, we derive the equations of motion of neutral molecules and their invariants, determine the conditions for these neutral molecules to become trapped in the field of the beams as a function of beam-pipe temperature, and compute the resulting enhancement of molecule density in the vicinity of the beam. We demonstrate that large agglomerates of molecules, "flakes," are much more likely to be pulled into the beam than single molecules, and suggest that this phenomenon might help explain some beam observations at the Large Hadron Collider.
\end{abstract}

DOI: 10.1103/PhysRevAccelBeams.24.054001

\section{INTRODUCTION}

During the 2017 and 2018 runs of the Large Hadron Collider (LHC), abnormal beam losses, some leading to emergency beam aborts, were observed at one particular location, in half-cell no. 16 left of LHC interaction point 2 ("16L2") [1,2]. These losses, occasionally accompanied by a fast beam instability [3], were later attributed to an accidental in-leak of air into the beam vacuum, which had occurred while the beam screen was at a temperature of $20 \mathrm{~K}$. This in-leak had resulted in a surface layer of frozen air molecules, including oxygen, nitrogen, and water [4,5]. Molecules of such a frozen surface layer could have been detached by beam-induced heating, synchrotron radiation,

\footnotetext{
*g.franchetti@gsi.de
}

Published by the American Physical Society under the terms of the Creative Commons Attribution 4.0 International license. Further distribution of this work must maintain attribution to the author(s) and the published article's title, journal citation, and DOI. or by the beam field. Detached molecules which approach the beam may interact with the circulating protons.

Molecules of oxygen or nitrogen are either paramagnetic or diamagnetic, respectively, having either a permanent or an induced magnetic dipole moment. Water molecules, on the other hand, carry a permanent electric dipole moment, which makes them "polar."

The electromagnetic force exerted on molecules exhibiting an induced magnetic or electric dipole moment is much weaker than the force experienced by paramagnetic or polar molecules, i.e., molecules with a permanent dipole moment. For this reason, in the following, we will not further discuss the motion of molecules with only induced dipole moments. Instead, our objective is to simulate and analyze the motion of neutral molecules carrying a permanent magnetic or electric dipole moment, in particular of the paramagnetic oxygen and of the polar water molecules, subjected to the charged-particle beam field.

If interactions with the residual gas limit the beam lifetime, the motion of neutral molecules inside the vacuum chamber becomes important. The motion of paramagnetic 
or polar molecules in an electromagnetic field is governed by their electric and magnetic dipole moments.

We start this article by surveying the electric and magnetic dipole moments of a few relevant molecules. Then we construct the equations of motion for such neutral molecules subjected to the electromagnetic field of the beam, at first crudely approximated by a thin-wire model. We solve these equations numerically, using a Runge-Kutta method, for different initial conditions, in order to identify the critical parameters for the molecule motion. Next, considering a more realistic Gaussian beam distribution, and recognizing important constants of motion, we estimate the position-dependent critical temperature for molecules to be trapped by the electric or magnetic field of the chargedparticle beam. Expanding this analytical treatment, we derive the general trapping efficiency as a function of temperature, and the local density enhancement resulting from the gas molecules' permanent dipole moments, which depends on beam current and temperature. Finally, we consider the special case of "flakes," i.e., agglomerates or clusters of many molecules carrying a dipole moment, and show that such flakes are more easily trapped in the field of the beam than their individual constituent molecules.

To simplify the equations, we are assuming the electric or magnetic dipole moments to always be aligned with the corresponding local field of the beam. In the Appendix we investigate the validity of this assumption.

\section{PERMANENT DIPOLE MOMENTS OF NEUTRAL MOLECULES}

The electric dipole moment (EDM) $\vec{p}$ for a pair of charges of the same magnitude but opposite sign is defined as

$$
\vec{p}=Q \vec{L},
$$

where $Q$ denotes the magnitude of charges and $\vec{L}$ is the displacement vector pointing from the negative charge to the positive charge. The SI unit of the EDM is the Coulomb meter $(\mathrm{C} \mathrm{m})$. In atomic physics also the unit Debye (D) is used $(1 \mathrm{D} \approx 0.21 e \AA$ with $e$ the electron charge and $1 \AA=0.1 \mathrm{~nm}$ ).

If a molecule with nonzero EDM is exposed to an external electric field the molecule experiences a torque $\vec{\tau}$ given by

$$
\vec{\tau}=\vec{p} \times \vec{E}
$$

Through the effect of $\tau$, the electric dipole of the molecule, hence the molecule itself, tends to align its axis with the direction of the applied electric field. The amount of the torque is maximum if the dipole moment is oriented at an angle of 90 degrees with respect to the external electric field.
Molecules with an asymmetric internal charge separation like $\mathrm{H}_{2} \mathrm{O}$ or $\mathrm{CO}$ feature a permanent nonzero EDM. In fact, we can decompose the dipole moment of $\mathrm{H}_{2} \mathrm{O}$, into an effective charge $Q$ and distance $L$, for example, as $Q=10 e$ and $|\vec{L}|=3.9 \times 10^{-12} \mathrm{~m}$, and hence $\left|\vec{p}_{\mathrm{H}_{2} \mathrm{O}}\right|=6.2 \times 10^{-30} \mathrm{Cm}$, where $e \approx 1.6 \times 10^{-19} \mathrm{C}$ denotes the elementary electron charge. For the water molecule, it should be noted that $L$ is a distance chosen to reproduce the measured dipole moment, for a given charge $Q$. However, the actual geometry of the $\mathrm{H}_{2} \mathrm{O}$ molecule resembles an isosceles triangle with two equal sides of about $1 \AA$ each, and one longer side of $1.6 \AA$. This circumstance should be carefully considered in future modeling. Similarly, for $\mathrm{CO}$, we can assign $Q=14 e$, and $|\vec{L}|=1.8 \times 10^{-13} \mathrm{~m}$, to obtain $\left|\vec{p}_{\mathrm{CO}}\right|=4.06 \times 10^{-31} \mathrm{C} \mathrm{m}$. On the other hand, molecules with a mirror-symmetric charge distribution, like $\mathrm{N}_{2}, \mathrm{O}_{2}$, or $\mathrm{CO}_{2}$, exhibit zero permanent EDM ([6,7] and Chapter 3 of [8]).

Like the electric dipole moment, also the magnetic dipole moment (MDM) $\vec{\mu}$ is known as a property of both fundamental and less fundamental particles. The MDM can be expressed as a vector $\vec{\mu}$,

$$
\vec{\mu}=\frac{e}{2 M} \eta \vec{v},
$$

where $e, M$ and $\eta$ are the charge, mass and reduced Planck constant of a particle, respectively. The unit vector $\vec{v}$ represents the direction of the MDM. In the case of the electron, $\vec{\mu}_{e}$ is known as a Bohr magneton (BM) and its value is $9.27 \times 10^{-24} \mathrm{~J} / \mathrm{T}$.

Molecules can also feature a nonzero MDM. Similar to the case of the EDM in an electric field, a molecule with a certain MDM exposed to an external magnetic field is subject to a torque $\vec{\tau}$, according to

$$
\vec{\tau}=\vec{\mu} \times \vec{B}
$$

For both EDM and MDM, the torque is zero only if the dipole moment axis is aligned with the field direction.

The magnitude of the molecular MDM depends on the electron configuration in the valence shell. The magnetic dipole moment arising due to unpaired electron spins is [9]

$$
\mu_{s}=\sqrt{n(n+2)} \mu_{e},
$$

where $n$ denotes the number of unpaired electrons. In the case of an oxygen molecule, $\mathrm{O}_{2}$, two unpaired electrons are present, $n=2$, so that the $\mu_{s}$ for oxygen is $2.8 \mu_{e}$. Due to this nonzero value of its MDM, the oxygen molecule exhibits paramagnetic properties.

Other molecules, like $\mathrm{N}_{2}, \mathrm{CO}_{2}, \mathrm{CO}$ and $\mathrm{H}_{2} \mathrm{O}$, do not possess any unpaired electron in their respective valence shell. Therefore, the spin magnetic dipole moment of these 
TABLE I. Permanent electric (EDM) and magnetic dipole moments (MDM) of various air molecules in units of Debye (D) and Bohr magneton (BM), respectively, along with the molecule mass $M$ in atomic mass units (amu).

\begin{tabular}{llcc}
\hline \hline Molecule & EDM [D] & MDM [BM] & $M$ [amu] \\
\hline $\mathrm{H}_{2} \mathrm{O}$ & 0.39 & 0 & 18 \\
$\mathrm{O}_{2}$ & 0 & 2.8 & 32 \\
$\mathrm{CO}$ & 0.025 & 0 & 28 \\
$\mathrm{~N}_{2}$ & 0 & 0 & 28 \\
$\mathrm{CO}_{2}$ & 0 & 0 & 44 \\
\hline \hline
\end{tabular}

molecules is zero $[6,8,10]$. These molecules are called diamagnetic.

In the case of an air in-leak into the vacuum system of an accelerator beam pipe, air molecules flowing into the vacuum pipe consist of nitrogen, oxygen, carbon dioxide, and water vapors, which carry specific electric and magnetic dipole moments. Table I shows relevant dipole moments for such air molecules, with their respective magnitude.

\section{MOLECULE MOTION ARISING FROM THE DIPOLE MOMENTS}

If a neutral molecule is exposed to an electric field $\vec{E}$ and to a magnetic field $\vec{B}$, its center of mass experiences the force

$$
\vec{F}_{t}=(\vec{\mu} \cdot \nabla) \vec{B}+(\vec{p} \cdot \nabla) \vec{E},
$$

where $\vec{p}$ and $\vec{\mu}$ denote the molecule's electric and magnetic dipole moment, respectively. In the following we assume that the magnetic and electric dipole moments are independent of location and, in particular, independent of the magnitude of the external fields.

As a next step, we now consider the force experienced by a molecule when subjected to the electric $\vec{E}$ and magnetic $\vec{B}$ field generated by the beam. As $\vec{p}$ and $\vec{\mu}$ oscillate around the direction of $\vec{E}$, and $\vec{B}$, respectively, the motion of molecules may become quite complex.

The dynamics of the molecules is characterized by multiple timescales. The shortest timescale corresponds to the vibrations of the atoms around their equilibrium position. The next, longer, timescale refers to the frequency at which the molecule's electric or magnetic dipole moment oscillates around the respective field. Lastly, the longest timescale refers to the periodicity of a molecule's centerof-mass motion under the influence of the field gradient.

If the period of oscillation of $\vec{p}$ and $\vec{\mu}$ is shorter than the characteristic time of change of $\vec{B}$ and $\vec{E}$ - this change being due either to the molecule dynamics or due the variation of the beam fields (individual bunch passages, injection, etc.)—we may assume that $\vec{p}$ and $\vec{\mu}$ are and remain aligned with their respective beam field. In the Appendix we present a detailed discussion on the validity of this approximation.

Our simplifying assumption would also imply a highly specific relation between $\vec{p}$ and $\vec{\mu}$, if both were simultaneously present, which, however, is not the case for any of the air molecules in question (compare Table I).

We note that in the case of a complete alignment of their magnetic and electric dipole moment with the corresponding beam field, the force on the molecules will be maximum, hence leading to the largest possible excursion of the molecule motion, and therefore, to a conservative assessment of the potential impact. In the Appendix, we examine the validity of the alignment assumption.

Without any other external field, or in close proximity to the beam, the dominant component of the electric or magnetic field is the one produced by the circulating beam.

In this section, we model the magnetic field by approximating the beam current as concentrated in a deltafunction-like manner into one transverse point at the center of the vacuum chamber, corresponding to the magnetic field of an extremely thin current-carrying wire. In later sections, we will consider the field of a more realistic beam distribution.

The thin-wire model properly describes the field at a radial distance $r$ outside of a round (or approximately round) beam. This field reads

$$
\vec{B}=\frac{\mu_{0} I}{2 \pi r} \frac{\vec{r}}{r} \times \hat{z},
$$

where $\hat{z}$ is a unit vector in the direction of the beam motion and $I$ denotes the average beam current. The quantity $\mu_{0}=$ $4 \pi \times 10^{-7} \mathrm{H} / \mathrm{m}$ is the permeability of free space. The motion of the neutral molecules typically is extremely slow compared with the time interval between the passage of individual bunches of the beam. For this reason, it is sufficient to consider the average beam current $I$, without taking into account any bunch structure. The magnetic field strength and field pattern are illustrated in Fig. 1.

Also the electric field generated by the round or approximately round beam, outside the beam core can approximately be described in a thin-wire approximation, as the field of a line charge distribution, namely as

$$
\vec{E}=\frac{\lambda}{2 \pi \epsilon_{0}} \frac{1}{r} \frac{\vec{r}}{r}
$$

where $\lambda=Q / C$ is the line charge density, with $Q$ the total charge in the accelerator and $C$ the accelerator circumference, $\epsilon_{0}=8.85 \times 10^{-12} \mathrm{~F} / \mathrm{m}$ the permittivity of free space, and $r$ the radial distance. For an ultrarelativistic beam we have $I=c \lambda$, and hence

$$
\vec{E}=\frac{I}{2 \pi \epsilon_{0} c} \frac{1}{r} \frac{\vec{r}}{r} .
$$




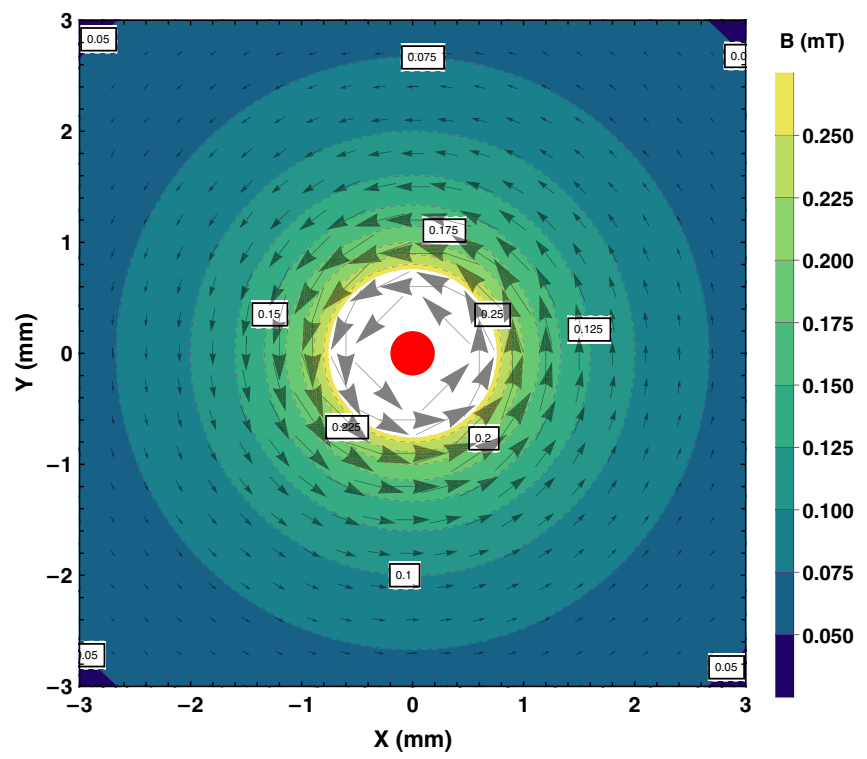

FIG. 1. Magnetic field line pattern around a beam. Magnetic field strength decreases as $1 / r$ with radial distance $r$ from the origin. The red spot at the center indicates the position of a proton beam leaving the picture plane towards the reader.

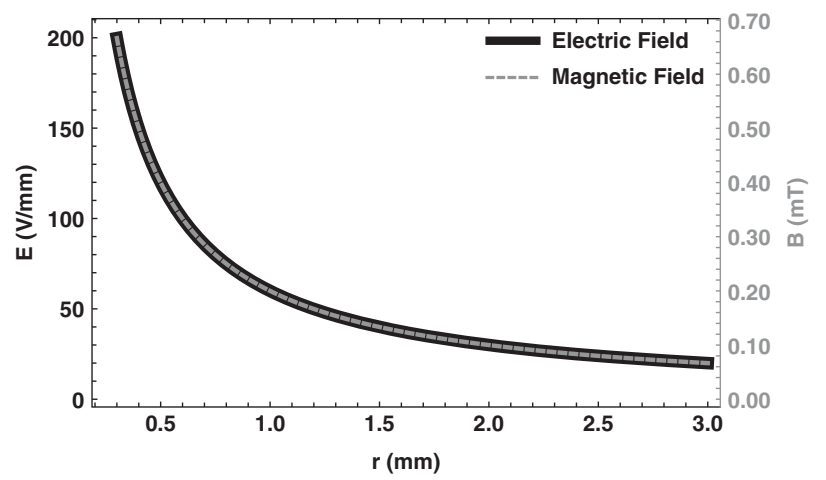

FIG. 2. Electric and magnetic field of the beam as a function of radial distance as generated by a pointlike thin-wire beam of current $1 \mathrm{~A}$.

Figure 2 displays the magnitude of the magnetic and electric fields for a beam with $I=1$ A current as a function of radial position and Fig. 3 illustrates the electric field pattern around the beam.

The equations of motion for molecules with electric and magnetic dipole moment are constructed under the approximation previously discussed. Namely we use our ansatz that the EDM and MDM are aligned with the electric and magnetic field generated by the beam, respectively. Namely we write

$$
\vec{p}=p \frac{\vec{r}}{r} \quad \text { and } \quad \vec{\mu}=\mu \frac{\vec{r}}{r} \times \hat{z}
$$

Inserting these equations, together with Eqs. (9) and (7), into Eq. (6), we obtain

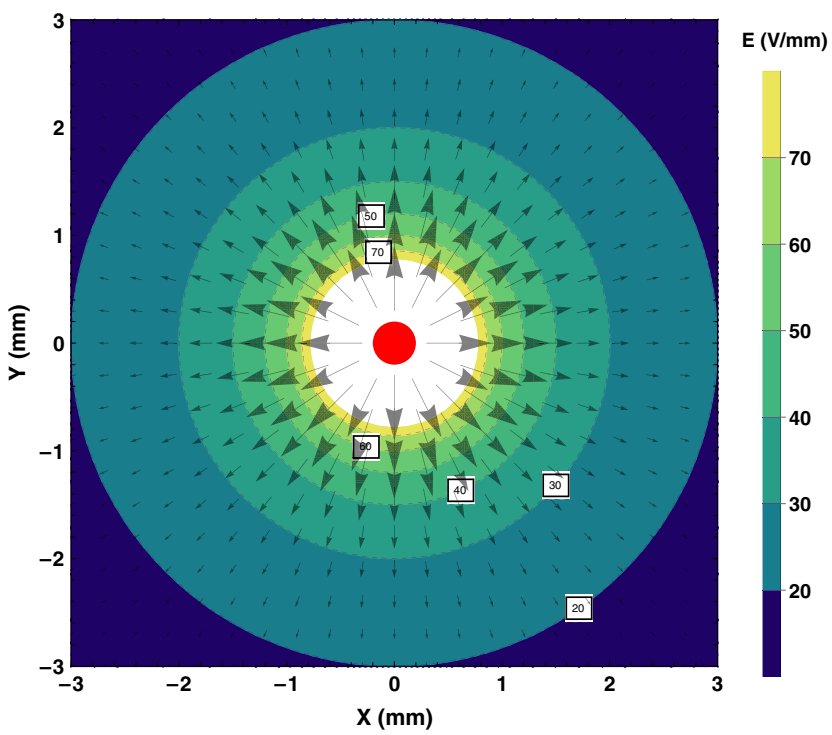

FIG. 3. Electric field lines around the round beam for a beam current of $1 \mathrm{~A}$.

$$
\begin{aligned}
& F_{x}=-\left(\frac{\mu \mu_{0} I}{2 \pi}+\frac{p I}{2 \pi \epsilon_{0} c}\right) \frac{x}{\left(x^{2}+y^{2}\right)^{3 / 2}}, \\
& F_{y}=-\left(\frac{\mu \mu_{0} I}{2 \pi}+\frac{p I}{2 \pi \epsilon_{0} c}\right) \frac{y}{\left(x^{2}+y^{2}\right)^{3 / 2}} .
\end{aligned}
$$

Hence, the equations of motion become

$$
\begin{aligned}
& \frac{d v_{x}}{d t}=-\frac{\tilde{p} I}{2 \pi \epsilon_{0} M c} \frac{x}{\left(x^{2}+y^{2}\right)^{3 / 2}}, \\
& \frac{d v_{y}}{d t}=-\frac{\tilde{p} I}{2 \pi \epsilon_{0} M c} \frac{y}{\left(x^{2}+y^{2}\right)^{3 / 2}},
\end{aligned}
$$

where $I$ is the beam current, $M$ is the molecule mass, and $\tilde{p}$ is an "effective" dipole moment, defined as

$$
\tilde{p}=p+\frac{\mu}{c}
$$

In order to integrate the equations of motion, the initial conditions of a molecule are required, namely its initial velocity and initial position, $\vec{r}_{0}$ and $\vec{v}_{0}$.

For our study we consider molecules having an initial velocity consistent with a certain temperature $T$, and having random component in the $x, y$ directions. The rms thermal transverse velocity of a molecule, in the $x-y$ plane, can be estimated by the classical formula,

$$
v_{\text {th }}=\sqrt{\frac{k_{b} T}{M}},
$$

where $k_{b}$ designates the Boltzmann constant, $T$ is the temperature and $M$ is the mass of the molecule. 

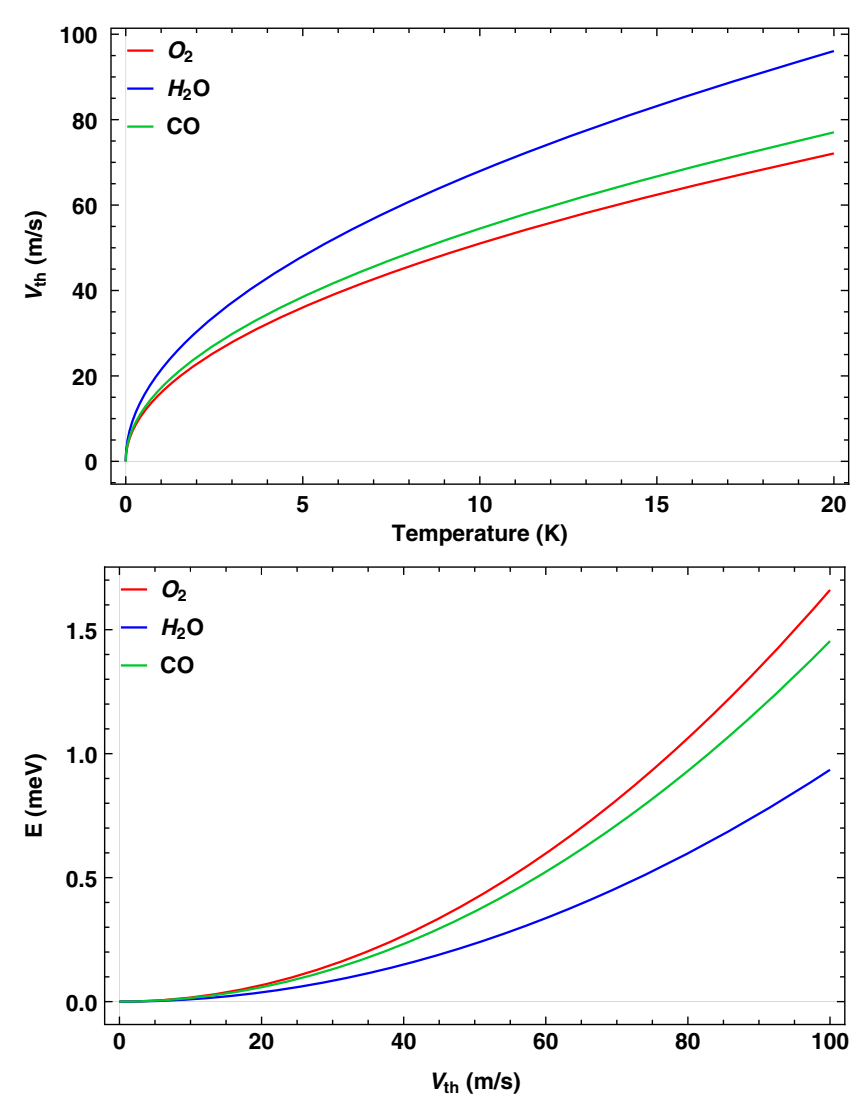

FIG. 4. The rms transverse thermal velocity of a molecule, $v_{\text {th }}$, as a function of temperature (top) and the average kinetic energy versus the rms thermal velocity $v_{\text {th }}$ (bottom).

The top panel of Fig. 4 represents the variation of $v_{\text {th }}$ as a function of temperature. The bottom panel shows the kinetic energy $E$ in units of $\mathrm{meV}$ as a function of $v_{\text {th }}$.

\section{MOTION PHENOMENOLOGY}

A numerical solution of the equations of motion has been implemented using the software package Mathematica [11]. SI units are used for all physical quantities. Different initial thermal velocities and positions are considered for the simulation of molecules motion in the electromagnetic field of the beam in order to identify the critical parameters. In the simulation we can vary, for example, the initial velocity and the initial position of a molecule.

The beam current is taken to be 1 Ampere. In all cases, we assume that a molecule's electric or magnetic dipole moment is always completely aligned with the electric or magnetic field of the beam, respectively (see the Appendix).

We first consider molecules at $1 \mathrm{~mm}$ distance from the beam with zero initial velocity. Such molecules will get trapped in the electromagnetic field of the beam and undergo an oscillatory motion. Figure 5 shows the horizontal motion of such $\mathrm{H}_{2} \mathrm{O}, \mathrm{CO}$, and $\mathrm{O}_{2}$ molecules, initially

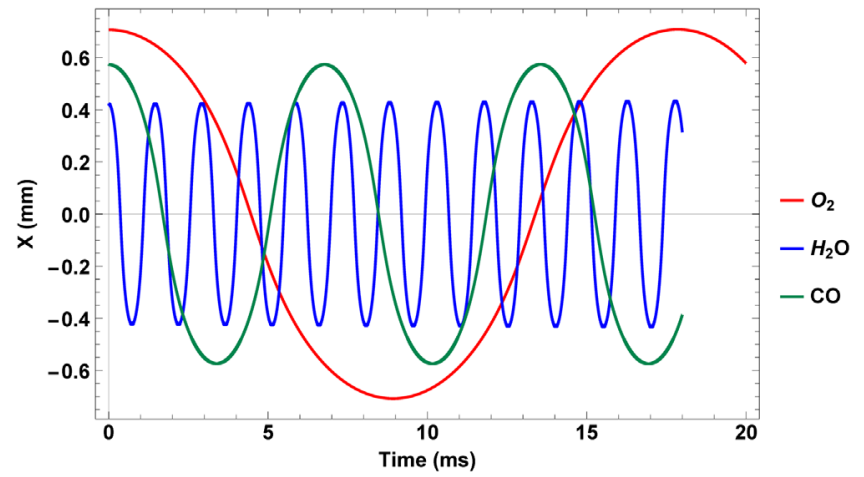

FIG. 5. Motion of neutral molecules initially $1 \mathrm{~mm}$ from the beam, at rest, and subjected to the electromagnetic field of the beam, as seen in the horizontal direction. Different colors correspond to different molecules as indicated.

at rest, as a function of time. The oscillation period depends on the dipole moment and on the mass of the molecules. In particular, the oxygen molecules perform oscillatory motion, if subjected to the magnetic field of the beam, due to their magnetic dipole moment, while they are not affected by the electric beam field, since they possess zero EDM. By contrast, water and carbon monoxide molecules undergo an oscillatory motion due to the electric field of the beam, since they carry a nonzero electric dipole moment. Figure 6 displays the molecule motion in the $x-y$ plane.

We now add an extremely small random initial velocity corresponding to a temperature of order $1 \mathrm{mK}$. Since particles are launched with different initial velocities in the $x$ and $y$ direction, the vertical and horizontal motion are not identical. Figure 7 presents some examples of $x$ and $y$ motion with respect to time, for this case of a small initial

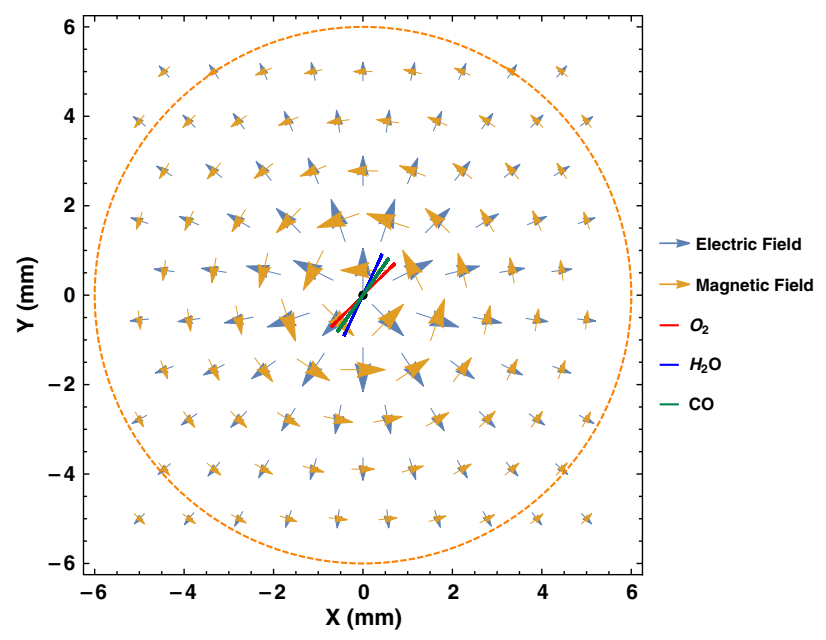

FIG. 6. The motion of neutral oxygen, water and carbon monoxide molecules initially at rest, $1 \mathrm{~mm}$ from the beam, and subjected to the electromagnetic field of a beam, in the transverse plane.With zero initial velocity, all molecules possessing either a magnetic or electric dipole moment undergo an oscillatory motion. 

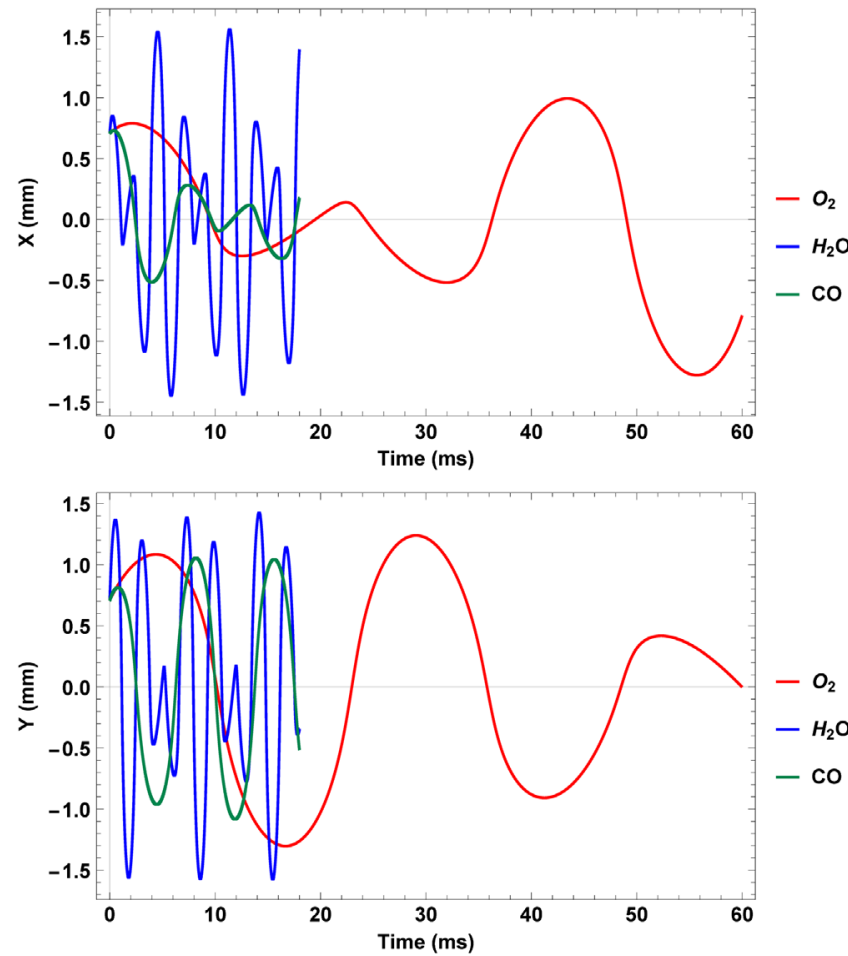

FIG. 7. Motion of neutral molecules initially at $1 \mathrm{~mm}$ from the beam with a thermal velocity corresponding to a temperature of $0.9 \mathrm{mK}$, and subjected to the electromagnetic field of the beam, in the horizontal (top) and the vertical direction (bottom). Different colors correspond to different molecules as indicated.

velocity, and Fig. 8 the corresponding picture of motion in the transverse plane.

We next increase the temperature to $15 \mathrm{~K}$, a typical temperature of the LHC beamscreen (which is held between about 5 and $20 \mathrm{~K}$ ). Molecules are again launched at a distance of $1 \mathrm{~mm}$ from the beam. Figures 9 and 10 present the molecule motion for this case. Under these conditions, single molecules of oxygen, water, and carbon monoxide do not get trapped in the electromagnetic field of the beam, as their large thermal velocity overcomes the effective potential created by the beam field. Instead of getting trapped in the beam field, these molecules hit the vacuum chamber wall. In our simple model here, we assume that the molecules then bounce back from the wall elastically. The wall is set at $6 \mathrm{~mm}$, about 3 times closer than the actual LHC beam pipe, which has a radius of about $20 \mathrm{~mm}$. Trapping at $15 \mathrm{~K}$ would still be possible if the initial distance of a molecule from the beam were just $1 \mathrm{~nm}(!)$.

Another possibility for molecules to be trapped is to increase their mass $M$, and, thereby, reduce their initial thermal velocity, according to Eq. (14). Indeed, it is extremely likely that molecules with a permanent dipole moment form flakes or clusters, consisting of 10,000 or many more oxygen or water molecules; see Sec. X. We should then explore the motion of such flakes. Again we

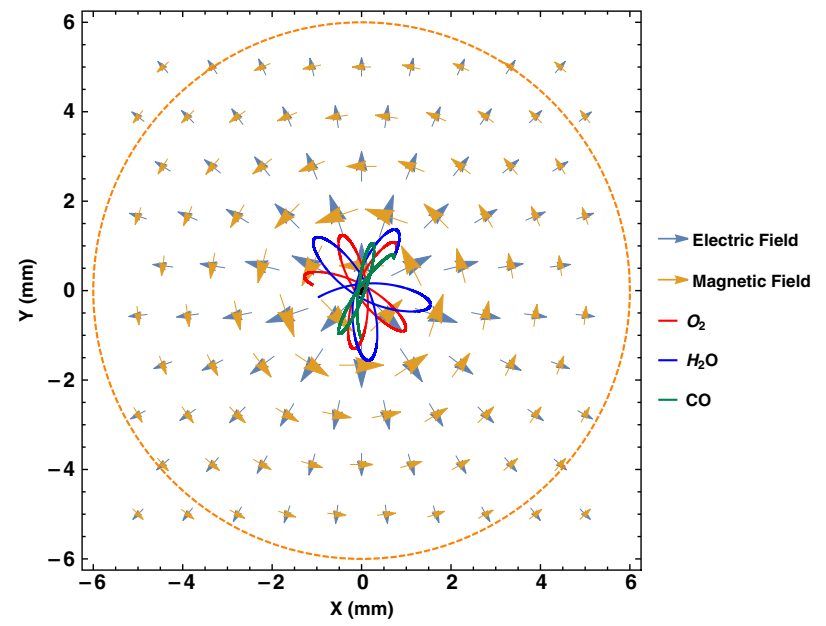

FIG. 8. The motion of neutral oxygen, water and carbon monoxide molecules initially at $1 \mathrm{~mm}$ from the beam with a thermal velocity corresponding to a temperature of order $1 \mathrm{mK}$, and subjected to the electromagnetic field of a beam, in the transverse plane. The initial thermal velocities were for $\mathrm{O}_{2} v_{\text {th }}=$ $0.2 \mathrm{~m} / \mathrm{s}(0.1 \mathrm{mK})$, for water $v_{\text {th }}=3 \mathrm{~m} / \mathrm{s}(20 \mathrm{mK})$, and for $\mathrm{CO}$ $v_{\text {th }}=0.3 \mathrm{~m} / \mathrm{s}(0.3 \mathrm{mK})$.

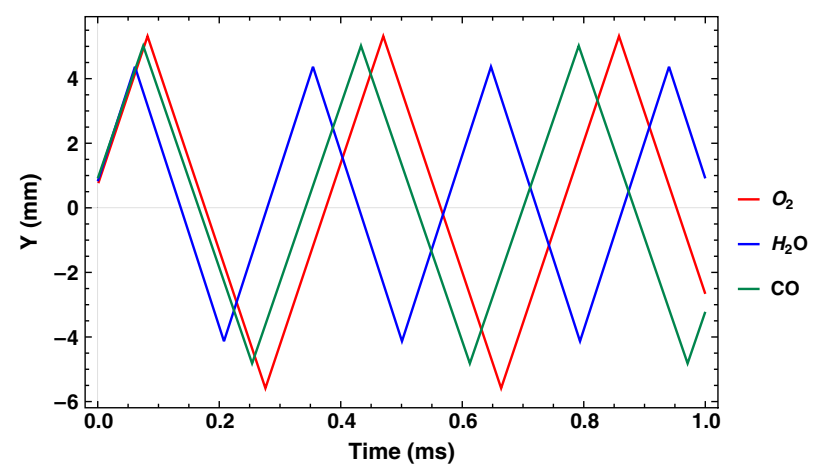

FIG. 9. Motion of neutral molecules initially at $1 \mathrm{~mm}$ from the beam with a thermal velocity corresponding to a temperature of $15 \mathrm{~K}$, and subjected to the electromagnetic field of the beam, in the vertical direction, with a hypothetical chamber wall at a radius of $6 \mathrm{~mm}$. Different colors correspond to different molecules as indicated.

may assume that the magnetic or electric dipole moment of a flake of molecules is completely aligned with the direction of the beam's electric or magnetic field. Our simple simulation shows that, in equilibrium at $15 \mathrm{~K}$, a flake of $10^{4}$ molecules will be captured by the beam field, even when its starting position is at a radial distance of $1 \mathrm{~mm}$. Figures 11 and 12 display the time evolution of $x$ and $y$ centroid coordinates and the corresponding phase space for a flake of molecules.

\section{TRAPPING BY BEAM POTENTIAL}

We observe that the dynamics described by the equations of motion (12) admit a constant of motion: 


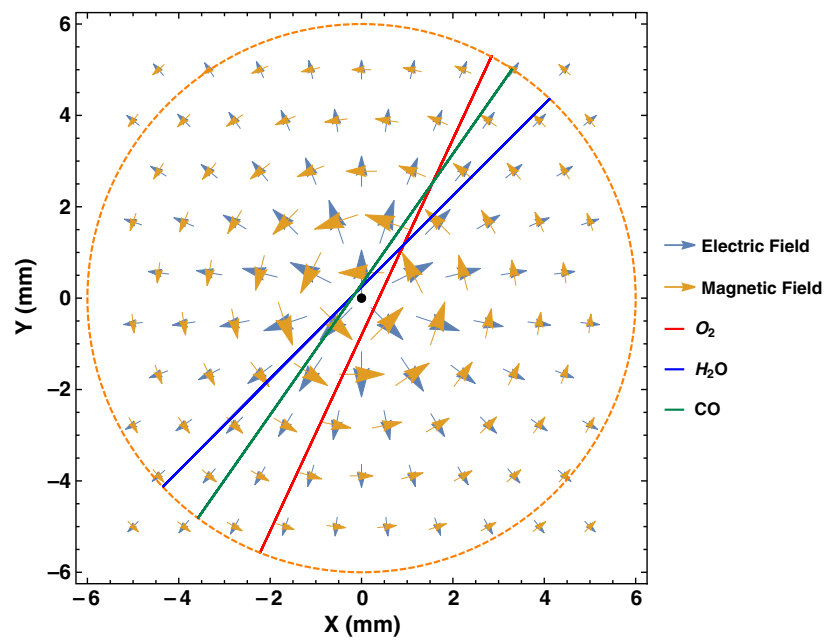

FIG. 10. The motion of neutral oxygen, water and carbon monoxide molecules initially at $1 \mathrm{~mm}$ from the beam with a thermal velocity corresponding to a temperature of $15 \mathrm{~K}$, and subjected to the electromagnetic field of a beam, in the transverse plane. The initial thermal velocities were $v_{\text {th }}=62 \mathrm{~m} / \mathrm{s}$ for $\mathrm{O}_{2}$, $v_{\text {th }}=82 \mathrm{~m} / \mathrm{s}$ for water, and $v_{\text {th }}=67 \mathrm{~m} / \mathrm{s}$ for CO. Note the assumption of perfect elastic collisions with the chamber wall.
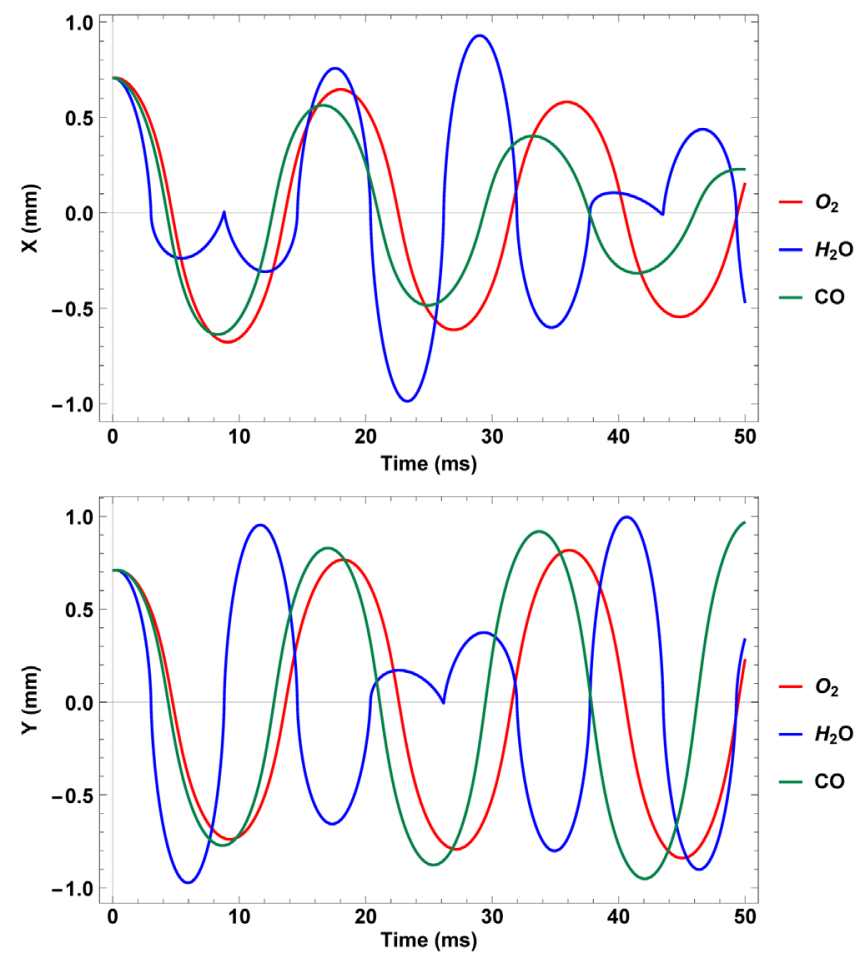

FIG. 11. Motion of neutral flakes of molecules initially at $1 \mathrm{~mm}$ from the thin-wire beam with a thermal velocity corresponding to a temperature of about $15 \mathrm{~K}$, and subjected to the electromagnetic field of the beam, in the horizontal (top) and the vertical direction (bottom). Different colors correspond to different types of constituent molecules as indicated.

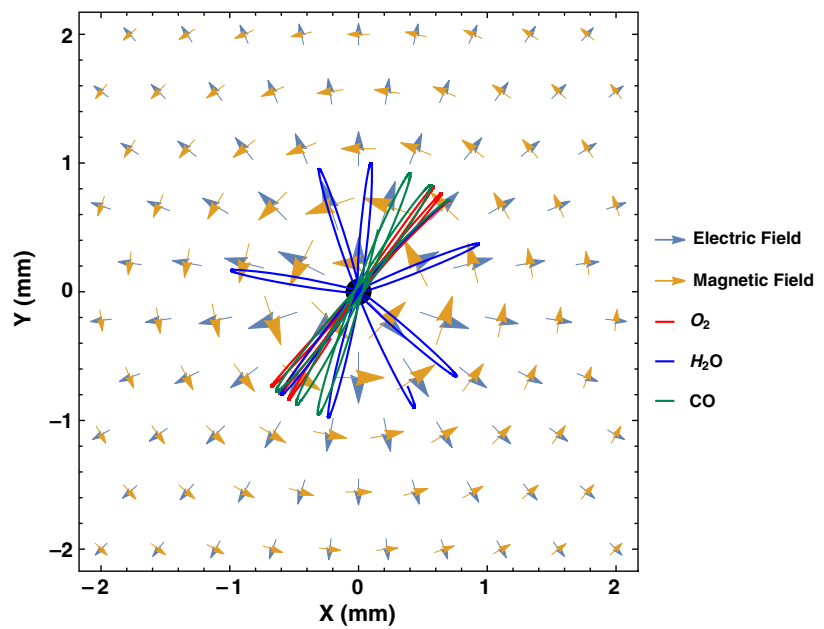

FIG. 12. The motion of neutral oxygen, water and carbon monoxide molecule flakes initially at rest, initially $1 \mathrm{~mm}$ from the thin-wire beam, and subjected to the electromagnetic beam field, in the transverse plane, for a pipe temperature of about $15 \mathrm{~K}$.

$$
C=\frac{1}{2}\left(v_{x}^{2}+v_{y}^{2}\right)-\frac{\tilde{p} I}{2 \pi \epsilon_{0} M c} \frac{1}{r} .
$$

If $C$ is positive the motion is unstable and the trajectory of the molecule diverges. If instead $C<0$ the motion is stable and a molecule oscillates between a maximum and a minimum radius. Therefore, for a molecule initially located at $\vec{r}_{0}$ and having a temperature $T_{0}$ defined by its initial velocities as $T_{0} \equiv M\left(v_{x, 0}^{2}+v_{y, 0}^{2}\right) / k_{b}$, the invariant $C$ assumes the value

$$
C=\frac{1}{2} \frac{k_{b} T_{0}}{M}-\frac{\tilde{p} I}{2 \pi \epsilon_{0} M c} \frac{1}{r_{0}} .
$$

In order for a molecule to exhibit stable motion (trapping), its invariant $C$ must be negative, and hence the temperature $T_{0}$ (in the following just written as $T$ ) must be smaller than a limiting temperature $T_{\lim }\left(r_{0}\right)$, according to

$$
T<T_{\lim }\left(r_{0}\right)=\frac{\tilde{p} I}{\pi \epsilon_{0} k_{b} c} \frac{1}{r_{0}} .
$$

Above the limiting temperature the molecule motion becomes unstable. Evidently, this condition depends on the initial radial position $r_{0}$. This formula also suggests that if $r_{0} \rightarrow 0$ any temperature is admitted, but this is an artifact of the thin-wire model for the beam, which we employ here. In fact, at $r \rightarrow 0$ the beam field diverges, which is nonphysical and originates from our adopted model. With this caveat, we can still roughly estimate a limiting temperature below which a molecule would be trapped. Such a limiting temperature can be obtained by considering an initial position at the beam pipe. Denoting by $R_{p}$ the radius of the beam pipe, we have 


$$
T_{\lim }=\frac{\tilde{p} I}{\pi \epsilon_{0} k_{b} c} \frac{1}{R_{p}} .
$$

However, even some molecules with $T<T_{\lim }$ may still be lost, and hit the beam pipe, even if they could not escape to infinity. By requiring that the maximum radius reached by a molecule be less than the beam pipe $R_{p}$, we can further constrain the set of initial positions of molecules with temperature $T$, in order for them to remain trapped by the beam field. With a little bit of algebra we find that a molecule with initial position $r_{0}$ and temperature $T$ is trapped inside the beam pipe, if

$$
\frac{r_{0}}{R_{p}} \leq \frac{1}{1+\frac{\pi \epsilon_{0} k_{b} c}{\tilde{p}} \frac{T R_{p}}{I}}=f .
$$

For an initially uniform distribution of molecules, the fraction of molecules trapped with respect to all molecules present in the beam pipe is $f^{2}$. Therefore, if $\tilde{n}$ signifies the density of the molecules at temperature $T$, the number of molecules trapped per unit length, i.e., the line density of trapped molecules, is

$$
\lambda_{\text {trapped }}=\tilde{n} f^{2} \pi R_{p}^{2} .
$$

Note that $\pi \epsilon_{0} k_{b} c=1.1 \times 10^{-25} \mathrm{C}^{2} /(\mathrm{Ks})$.

For example, considering $\mathrm{H}_{2} \mathrm{O}$ molecules, with $\tilde{p} \simeq$ $6.2 \times 10^{-30} \mathrm{C} \mathrm{m}$, we find

$$
f=\frac{1}{1+18.5 \times 10^{3} \frac{T R_{p}}{I}} .
$$

Further, with $I=1 \mathrm{~A}$, and $R_{p}=0.1 \mathrm{~m}$ the expression for $f$ becomes

$$
f=\frac{1}{1+1.85 \times 10^{3} T[K]},
$$

which implies that only extremely cold molecules can be trapped by the beam. At $T=54 \mathrm{mK}$ the normalized trapping radius would be $f=0.5$, and, consequently, $25 \%$ of the molecules of this temperature present in the pipe would be trapped. If instead we require that a molecule trapped remains inside the beam of radius $R_{b}=3 \sigma$ (with $\sigma$ the true rms beam size), then we need to demand

$$
\frac{r_{0}}{R_{b}} \leq \frac{1}{1+\frac{\pi \epsilon_{0} k_{b} c}{\tilde{p}} \frac{T R_{b}}{I}} .
$$

For $\sigma=0.333 \mathrm{~mm}$, we find $R_{b}=1 \mathrm{~mm}$ and

$$
\frac{r_{0}}{3 \sigma} \leq \frac{1}{1+18.5 T[K]} .
$$

Therefore, if $T=0.108 \mathrm{~K}$, a molecule initially at $r_{0} / \sigma \leq 1$ will be trapped within the radius $R_{b}=3 \sigma$. Basically, the hotter molecules have to be closer to the beam center, where the fields are stronger, in order to be trapped. Conversely, molecules at a distance $r_{0}=R_{b}=3 \sigma$ would need to be extremely cold to remain inside the beam. This, however, is an artifact of the adopted thin-wire model for the beam field. A more realistic model will be developed in the next section.

\section{ARBITRARY AXISYMMETRIC BEAM}

Next we consider an axisymmetric beam with charge line distribution,

$$
\rho(r)=\frac{I}{\pi \sigma^{2} c} \mathrm{n}\left(\frac{r^{2}}{\sigma^{2}}\right),
$$

defined for an ultrarelativistic beam, with $\mathrm{n}(u)$ a function of $u$ satisfying the normalization

$$
\int_{0}^{\infty} \mathrm{n}(u) d u=1 .
$$

For a Gaussian distribution, this function assumes the form

$$
\mathrm{n}(u)=\frac{1}{2} e^{-\frac{u}{2}}
$$

By applying Gauss' law, we find the electric field $\vec{E}(\vec{r})=E(r) \vec{r} / r$, with

$$
E(r)=\frac{1}{2 \pi \epsilon_{0} c} I \frac{1}{r} \mathrm{~N}\left(\frac{r^{2}}{\sigma^{2}}\right),
$$

where for convenience we define the function

$$
\mathrm{N}(u)=\int_{0}^{u} \mathrm{n}\left(u^{\prime}\right) d u^{\prime} .
$$

The magnetic field is computed by applying Ampere's law, yielding

$$
\vec{B}(\vec{r})=B(r) \frac{\vec{r}}{r} \times \hat{z},
$$

with

$$
B(r)=\frac{\mu_{0}}{2 \pi} I \frac{1}{r} \mathrm{~N}\left(\frac{r^{2}}{\sigma^{2}}\right) .
$$

Next we apply Eq. (6) to the magnetic field case for an aligned MDM to obtain

$$
(\vec{\mu} \cdot \nabla) \vec{B}=\mu \sum_{i}\left(\frac{\vec{r}}{r} \times \hat{z}\right)_{i} \partial_{i}\left[B(r) \frac{\vec{r}}{r} \times \hat{z}\right] .
$$


With some straightforward algebra we obtain

$$
(\vec{\mu} \cdot \nabla) \vec{B}=-\mu \frac{B(r)}{r} \frac{\vec{r}}{r} .
$$

Instead, for the electric field, and, as before, considering the EDM to be aligned with the field, the force on the molecule becomes

$$
(\vec{p} \cdot \nabla) \vec{E}=p \sum_{i}\left(\frac{\vec{r}}{r}\right)_{i} \partial_{i}\left[E(r) \frac{\vec{r}}{r}\right]
$$

which, again with straightforward algebra, becomes

$$
(\vec{p} \cdot \nabla) \vec{E}=p E^{\prime}(r) \frac{\vec{r}}{r}
$$

Therefore, the general force acting on a molecule exposed to the electric and magnetic field of an axisymmetric beam is

$\vec{F}_{t}=(\vec{p} \cdot \nabla) \vec{E}+(\vec{\mu} \cdot \nabla) \vec{B}=\left[p E^{\prime}(r)-\mu \frac{B(r)}{r}\right] \frac{\vec{r}}{r}$.

Now we substitute the expressions for $E(r)$ and $B(r)$ to find

$$
\vec{F}_{t}=\frac{1}{2 \pi \epsilon_{0} c} I\left\{p\left[\frac{1}{r} \mathrm{~N}\left(\frac{r^{2}}{\sigma^{2}}\right)\right]^{\prime}-\frac{\mu}{c} \frac{1}{r^{2}} \mathrm{~N}\left(\frac{r^{2}}{\sigma^{2}}\right)\right\} \frac{\vec{r}}{r},
$$

that is

$$
\vec{F}_{t}=\frac{1}{2 \pi \epsilon_{0} c} \frac{I}{\sigma^{2}}\left[2 p \mathrm{n}\left(\frac{r^{2}}{\sigma^{2}}\right)-\tilde{p} \frac{\sigma^{2}}{r^{2}} \mathrm{~N}\left(\frac{r^{2}}{\sigma^{2}}\right)\right] \frac{\vec{r}}{r},
$$

with the previous definition of the effective dipole moment, $\tilde{p}=(p+\mu / c)$ [see Eq. (13)].

We note that for particles outside a beam of rms size $\sigma$, namely at $r \gg \sigma$, we have $\mathrm{n}\left(r^{2} / \sigma^{2}\right) \approx 0$ and $\mathrm{N}\left(r^{2} / \sigma^{2}\right) \approx 1$, so that outside the beam the formula for the force equals the one of the thin-wire model.

In Eq. (31), the term in square brackets is a geometric term, specific to the type of beam distribution. If in the core of the beam the radial charge density is uniform, we expect

$$
\mathrm{n}(u) \approx n_{0} \quad \text { and } \quad \mathrm{N}(u) \approx n_{0} u,
$$

with $n_{0}=\mathrm{n}(0)$, so that Eq. (31) reads

$$
\vec{F}_{t} \approx \frac{1}{2 \pi \epsilon_{0} c} \frac{I}{\sigma^{2}} n_{0}[2 p-\tilde{p}] \frac{\vec{r}}{r}
$$

and the force for the EDM is of opposite sign to the one for the MDM.
For the EDM, we obtain

$$
\vec{F}_{t} \approx \frac{1}{2 \pi \epsilon_{0} c} \frac{I}{\sigma^{2}} n_{0} p \frac{\vec{r}}{r}
$$

while, for the MDM, we have

$$
\vec{F}_{t} \approx-\frac{1}{2 \pi \epsilon_{0} c} \frac{I}{\sigma^{2}} n_{0} \frac{\mu}{c} \frac{\vec{r}}{r}
$$

So, it seems that molecules with EDM cannot be trapped in the core of the beam where the beam density is uniform. This situation is unusual, because, once EDM molecules leave the beam core, they experience a stabilizing force, corresponding to the force of the thin-wire model.

Figure 13 illustrates the horizontal forces experienced by molecules with either an EDM or a MDM, as a function of the horizontal position $x$ along an axis passing through the center of the Gaussian beam, located at $x=0$. For convenience, the forces [Eq. (31)] are normalized by multiplying with $(2 \sigma) /\left(k_{b} T_{p}^{*}\right)$ or $(2 \sigma) /\left(k_{b} T_{\mu}^{*}\right)$, where we define the reference temperature

$$
T_{p}^{*}=\frac{1}{\pi \epsilon_{0} k_{b} c} \frac{I}{\sigma} p,
$$

or, respectively,

$$
T_{\mu}^{*}=\frac{1}{\pi \epsilon_{0} k_{b} c} \frac{\mu}{c} \frac{I}{\sigma} .
$$

We will refer to $T_{p}^{*}$, Eq. (33), and $T_{\mu}^{*}$, Eq. (34), as the "trapping temperature." The shape of the force in Fig. 13 suggests that the EDM molecules may oscillate not around the beam center, but rather around an equilibrium radius $r_{e}$, which is of the order of the beam size.

Incidentally, the trapping temperatures $T_{p}^{*}$ and $T_{\mu}^{*}$ resemble the limiting temperature $T_{\text {lim }}\left(r_{0}\right)$ defined in Eq. (16) or $T_{\lim }$ of Eq. (17). The various characteristic temperatures simply correspond to different areas of trapping. While $T_{p}^{*}$ and $T_{\mu}^{*}$ refer to trapping over a radial length scale equal to the rms beam size, the temperature $T_{\text {lim }}$ indicates trapping within the beam pipe $R_{p}$, for the thinwire model. A universal trapping temperature could be introduced as $T^{*}=\tilde{p} I /\left(\pi \epsilon_{0} k_{b} c S\right)$, with $S$ denoting the characteristic length scale of trapping.

In general, from (31), the equilibrium radius $r_{e}$ for the $\mathrm{EDM}$ is the solution of the equation

$$
2 \mathrm{n}\left(\frac{r^{2}}{\sigma^{2}}\right)=\frac{\sigma^{2}}{r^{2}} \mathrm{~N}\left(\frac{r^{2}}{\sigma^{2}}\right) .
$$

For a Gaussian beam 


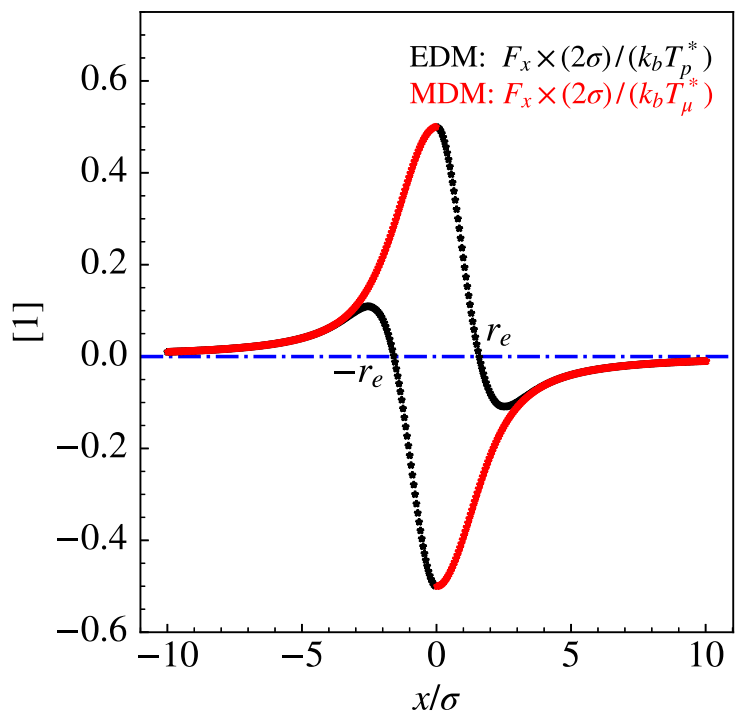

FIG. 13. The horizontal normalized force acting on an EDM (black) or MDM molecule (red), respectively, as a function of the transverse $(x, 0)$ coordinate. The equilibrium position for the EDM case is indicated by the label $r_{e}$. Note that for both types of dipole moment the force is discontinuous at $x=0$.

$$
\mathrm{n}(u)=\frac{1}{2} e^{-\frac{u}{2}}, \quad \mathrm{~N}(u)=\int_{0}^{u / 2} e^{-w} d w=1-e^{-u / 2},
$$

so that Eq. (35) becomes

$$
w^{2} e^{-w^{2} / 2}=1-e^{-w^{2} / 2}
$$

with $w^{2}=r_{e}^{2} / \sigma^{2}$. Its solution is $r_{e} / \sigma=1.585201 \simeq \pi / 2$.

For a beam with a Kapchinskij-Vladimirskij (K-V) distribution [12,13], instead, such equilibrium solution does not exist, and the force acting on an EDM molecule will be subject to a discontinuity at the beam edge. Namely, for a $\mathrm{K}-\mathrm{V}$ beam, at $r<R_{b}$ the force is constant and defocusing, whereas at $r>R_{b}$ it is attractive and nonlinearly decreasing with amplitude.

\section{OSCILLATION FREQUENCY}

According to (31), and introducing the radial velocity $\vec{v} \equiv d \vec{r} / d t$, for EDM molecules the equation of motion reads

$$
\frac{d \vec{v}}{d t}=\frac{1}{2 \pi \epsilon_{0} M c} \frac{I}{\sigma^{2}} p\left[2 \mathrm{n}\left(\frac{r^{2}}{\sigma^{2}}\right)-\frac{\sigma^{2}}{r^{2}} \mathrm{~N}\left(\frac{r^{2}}{\sigma^{2}}\right)\right] \frac{\vec{r}}{r} .
$$

Expanding around the equilibrium radius $r_{e}$ we find

$$
\frac{d \vec{v}}{d t}=\frac{1}{2 \pi \epsilon_{0} M c} \frac{I}{\sigma^{2}} p\left[4 \frac{r_{e}}{\sigma} \mathrm{n}^{\prime}\left(\frac{r_{e}^{2}}{\sigma^{2}}\right)+2 \frac{\sigma}{r_{e}} \mathrm{n}\left(\frac{r_{e}^{2}}{\sigma^{2}}\right)\right] \frac{r-r_{e}}{\sigma} \frac{\vec{r}}{r} .
$$

Using the equilibrium radius $r_{e}$ and $\mathrm{n}(u)$ for a Gaussian distribution, to good approximation, the term in the square bracket is equal to $-e / 10$ (the exact number is $-0.2716776662 \ldots)$. Then Eq. (37) becomes

$$
\frac{d \vec{v}}{d t} \approx-\frac{e}{10} \frac{p}{2 \pi \epsilon_{0} M c} \frac{I}{\sigma^{2}} \frac{\left(r-r_{e}\right)}{\sigma} \frac{\vec{r}}{r} .
$$

Therefore, the angular oscillation frequency is

$$
\omega \approx \sqrt{\frac{e}{10} \frac{p}{2 \pi \epsilon_{0} M c \sigma^{3}} I .}
$$

For a water molecule, $\mathrm{H}_{2} \mathrm{O}$, characterized by $M=$ $3 \times 10^{-26} \mathrm{~kg}$ and $p=6.2 \times 10^{-30} \mathrm{Cm}$, and a beam with $\sigma=3 \times 10^{-4} \mathrm{~m}$ and $I=1 \mathrm{~A}$, we find $\omega=11189 \mathrm{rad} / \mathrm{s}$. Hence, in this case, the frequency of oscillation around the equilibrium radius $r_{e}$ is $f=\omega /(2 \pi)=1780 \mathrm{~Hz}$, which, for the LHC, is of the order of the fractional betatron frequency.

For a molecule featuring an MDM, instead of Eq. (38) we have

$$
\frac{d \vec{v}}{d t}=-\frac{1}{2 \pi \epsilon_{0} M c} \frac{I}{\sigma^{2}} \frac{\mu}{c} \frac{\sigma^{2}}{r^{2}} \mathrm{~N}\left(\frac{r^{2}}{\sigma^{2}}\right) \frac{\vec{r}}{r} .
$$

Now the equilibrium radius is the beam center $r=0$. Close to this point, with a central beam density $n_{0}$, the oscillatory motion follows the equation

$$
\frac{d \vec{v}}{d t}=-\frac{1}{2 \pi \epsilon_{0} M c} \frac{I}{\sigma^{2}} \frac{\mu}{c} n_{0} \frac{\vec{r}}{r},
$$

and so the beam seems to create a constant central force pointing towards the beam center. Here, an MDM molecule oscillates at a frequency which depends on the square root of the maximum oscillation amplitude.

\section{INVARIANTS AND TRAPPING}

Starting again from (31) and multiplying with the radial velocity $\vec{v} \equiv v \vec{r} / r$, we can rewrite the equation of motion as

$$
\begin{aligned}
& \frac{d}{d t} \frac{1}{2} v^{2} \\
& =\frac{1}{2 \pi \epsilon_{0} M c} \frac{I}{\sigma}\left[p \frac{\sigma}{r} \frac{d}{d(r / \sigma)} \mathrm{N}\left(\frac{r^{2}}{\sigma^{2}}\right)-\tilde{p} \frac{\sigma^{2}}{r^{2}} \mathrm{~N}\left(\frac{r^{2}}{\sigma^{2}}\right)\right] \frac{d}{d t}\left(\frac{r}{\sigma}\right) .
\end{aligned}
$$

For EDM molecules we have

$$
\frac{d}{d t} \frac{1}{2} v^{2}=\frac{1}{2 \pi \epsilon_{0} M c} \frac{I}{\sigma} p \frac{d}{d t}\left[\frac{\sigma}{r} \mathrm{~N}\left(\frac{r^{2}}{\sigma^{2}}\right)\right],
$$

and the integral of motion becomes 


$$
\frac{1}{2} v^{2}-\frac{k_{b} T_{p}^{*}}{2 M} \frac{\sigma}{r} \mathrm{~N}\left(\frac{r^{2}}{\sigma^{2}}\right)=D
$$

where we have used the reference temperature $T_{p}^{*}$, defined in Eq. (33). The constant $D$ is determined by the initial conditions, namely

$$
\frac{1}{2} v_{0}^{2}-\frac{k_{b} T_{p}^{*}}{2 M} \frac{\sigma}{r_{0}} \mathrm{~N}\left(\frac{r_{0}^{2}}{\sigma^{2}}\right)=D
$$

Now, the condition for a particle with initial conditions $\left(r_{0}, v_{0}\right)$ to be trapped within the radius $R_{b}$ is

$$
v_{0}^{2}<\frac{k_{b} T_{p}^{*}}{M}\left[\frac{\sigma}{r_{0}} \mathrm{~N}\left(\frac{r_{0}^{2}}{\sigma^{2}}\right)-\frac{\sigma}{R_{b}} \mathrm{~N}\left(\frac{R_{b}^{2}}{\sigma^{2}}\right)\right] .
$$

Note that not all values of $r_{0}$ are admitted. In fact, for $r_{0} \rightarrow 0$ we find

$$
\frac{\sigma}{r_{0}} \mathrm{~N}\left(\frac{r_{0}^{2}}{\sigma^{2}}\right) \rightarrow 0
$$

Therefore, as could be guessed by looking at Fig. 13, there is a minimum $r_{0, \min }$ associated with $R_{b}$ so that

$$
\frac{\sigma}{r_{0, \min }} \mathrm{N}\left(\frac{r_{0, \min }^{2}}{\sigma^{2}}\right)=\frac{\sigma}{R_{b}} \mathrm{~N}\left(\frac{R_{b}^{2}}{\sigma^{2}}\right)
$$

Molecules with a smaller initial value of $r_{0}$ cannot be trapped.

Equation (45) sets a constraint on the trapping for a gas of molecules of temperature $T$. At a given value of $r_{0}$, the fraction $f$ of the particles that can be trapped is obtained by integrating the Maxwell-Boltzmann distribution:

$$
f=\int_{v_{x}^{2}+v_{y}^{2}<v_{0}^{2}} \frac{1}{2 \pi} \frac{M}{k_{b} T} e^{-\frac{M v^{2}}{2 k_{b} T}} d v_{x} d v_{y}=1-\exp \left(-\frac{1}{2} \frac{M v_{0}^{2}}{k_{b} T}\right)
$$

as

$f=1-\exp \left\{-\frac{1}{2} \frac{T_{p}^{*}}{T}\left[\frac{\sigma}{r_{0}} \mathrm{~N}\left(\frac{r_{0}^{2}}{\sigma^{2}}\right)-\frac{\sigma}{R_{b}} \mathrm{~N}\left(\frac{R_{b}^{2}}{\sigma^{2}}\right)\right]\right\}$.

The total amount of gas trapped in the beam at $R_{b}=3 \sigma$ is determined by integrating over the initial radii:

$$
\begin{aligned}
N_{\text {trap }}= & \tilde{n} \int_{r_{0, \text { min }}}^{R_{b}}\left(1-\exp \left\{-\frac{1}{2} \frac{T_{p}^{*}}{T}\left[\frac{\sigma}{r_{0}} \mathrm{~N}\left(\frac{r_{0}^{2}}{\sigma^{2}}\right)\right.\right.\right. \\
& \left.\left.\left.-\frac{\sigma}{R_{b}} \mathrm{~N}\left(\frac{R_{b}^{2}}{\sigma^{2}}\right)\right]\right\}\right) 2 \pi r_{0} d r_{0},
\end{aligned}
$$

where $\tilde{n}$ denotes the gas density (molecules per volume). This can be further transformed to
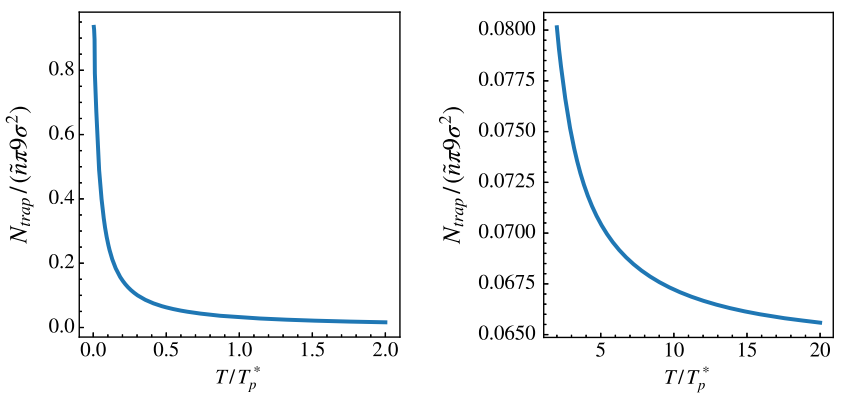

FIG. 14. Fraction of EDM molecules trapped in the beam as a function of $T / T_{p}^{*}$, for $T$ from 0 to $2 T_{p}^{*}$ (left) and through $20 T_{p}^{*}$ (right). For this calculation we considered trapping inside a radius of $R_{b}=3 \sigma$, with $\sigma$ the rms beam size.

$$
\begin{aligned}
\frac{N_{\text {trap }}}{\tilde{n} \pi R_{b}^{2}}= & 1-\left(\frac{r_{0, \min }}{R_{b}}\right)^{2}-2 \frac{\sigma^{2}}{R_{b}^{2}} \exp \left[\frac{1}{2} \frac{T_{p}^{*}}{T} \frac{\sigma}{R_{b}} \mathrm{~N}\left(\frac{R_{b}^{2}}{\sigma^{2}}\right)\right] \\
& \times \int_{r_{0, \min } / \sigma}^{R_{b} / \sigma} \exp \left[-\frac{1}{2} \frac{T_{p}^{*}}{T} \frac{\mathrm{N}\left(u^{2}\right)}{u}\right] u d u .
\end{aligned}
$$

This formula can be integrated numerically with results shown in Fig. 14, for the case of a Gaussian distribution.

For $\mathrm{H}_{2} \mathrm{O}$ with a beam of $1 \mathrm{~A}$ beam current and $\sigma=3 \times 10^{-4} \mathrm{~m} \mathrm{rms}$ beam size we find $T_{p}^{*}=0.18 \mathrm{~K}$. This temperature is of similar magnitude as the trapping temperature predicted earlier by the thin-wire model, but the trapping efficiency is much reduced for this more realistic Gaussian beam distribution. Indeed, the right panel of Fig. 14 suggests that for a gas of $\mathrm{H}_{2} \mathrm{O}$ molecules with temperature $T=2 \mathrm{~K}$, corresponding to $T / T_{p}^{*}=11.1$, the fractional trapping is $\simeq 0.016$, or only about $1.6 \%$. If instead $T=T_{p}^{*}$, Fig. 14 (right) shows that the fraction of trapped particles would be $\simeq 0.067$ or close to $7 \%$.

For MDM molecules, the equation of motion becomes

$$
\frac{d}{d t} \frac{1}{2} v^{2}=-\frac{1}{2 \pi \epsilon_{0} M c} \frac{I}{\sigma} \frac{\mu}{c} \frac{d}{d t} \int_{0}^{r / \sigma} \frac{1}{u^{2}} \mathrm{~N}\left(u^{2}\right) d u,
$$

yielding the constant of motion $E$,

$$
\frac{1}{2} v^{2}+\frac{k_{b} T_{\mu}^{*}}{2 M} \mathrm{~N}_{2}\left(\frac{r}{\sigma}\right)=E
$$

where we have used the trapping temperature $T_{\mu}^{*}$, defined in Eq. (34), and the new function

$$
\mathrm{N}_{2}(u)=\int_{0}^{u} \frac{1}{v^{2}} \mathrm{~N}\left(v^{2}\right) d v
$$

In this case, the motion may be confined for $E>0$. In particular, for a molecule to be trapped inside the beam radius $R_{b}$, its phase-space coordinates $r, v$ should satisfy 


$$
v^{2}+\frac{k_{b} T_{\mu}^{*}}{M} \mathrm{~N}_{2}\left(\frac{r}{\sigma}\right) \leq \frac{k_{b} T_{\mu}^{*}}{M} \mathrm{~N}_{2}\left(\frac{R_{b}}{\sigma}\right)
$$

or

$$
v^{2} \leq \frac{k_{b} T_{\mu}^{*}}{M}\left[\mathrm{~N}_{2}\left(\frac{R_{b}}{\sigma}\right)-\mathrm{N}_{2}\left(\frac{r}{\sigma}\right)\right]
$$

In this case the fraction of trapped particles in a Maxwellian gas is

$$
f=1-\exp \left\{-\frac{1}{2} \frac{T_{\mu}^{*}}{T}\left[\mathrm{~N}_{2}\left(\frac{R_{b}}{\sigma}\right)-\mathrm{N}_{2}\left(\frac{r}{\sigma}\right)\right]\right\} .
$$

As before, this result applies to particles located at $r / \sigma$. Again, the total fraction of trapped particles is obtained by integrating over all locations in the beam pipe, that is

$$
\begin{aligned}
\frac{N_{\text {trap }}}{\tilde{n} \pi R_{b}^{2}}= & 1-2 \frac{\sigma^{2}}{R_{b}^{2}} \exp \left[-\frac{1}{2} \frac{T_{\mu}^{*}}{T} \mathrm{~N}_{2}\left(\frac{R_{b}}{\sigma}\right)\right] \\
& \times \int_{0}^{R_{b} / \sigma} \exp \left(\frac{1}{2} \frac{T_{\mu}^{*}}{T} \mathrm{~N}_{2}(u)\right) u d u .
\end{aligned}
$$

Figure 15 displays the total fraction of trapped molecules as a function of $T / T_{\mu}^{*}$ for the case of a beam with an axisymmetric Gaussian distribution.

As an example, considering oxygen molecules, $\mathrm{O}_{2}$, an rms beam size $\sigma=3 \times 10^{-4} \mathrm{~m}$ and an average beam current $I=1 \mathrm{~A}$, we find $T_{\mu}^{*}=2.5 \mathrm{mK}$. So, for molecules at a temperature of $T=2 \mathrm{~K}$, we have $T / T_{\mu}^{*}=800$ and the fraction of trapped molecules is only $1.21 \times 10^{-4}$ or $0.0121 \%$, while for $T / T_{\mu}^{*}=1$ the fractional trapping is $\simeq 0.088$ or about $8.8 \%$.

\section{PINCH OF NEUTRAL MOLECULES}

The fraction of trapped molecules, extracted from the constant of motion and the temperature, is an important indicator, but it does not provide any prediction for the average particle density as a function of radial position.
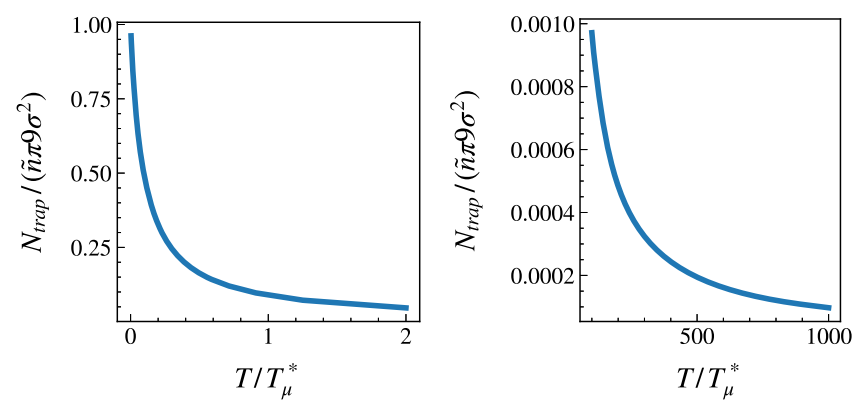

FIG. 15. Fraction of MDM molecules trapped in the beam as a function of $T / T_{\mu}^{*}$, for $T$ from 0 to $2 T_{\mu}^{*}$ (left) and through $1000 T_{\mu}^{*}$ (right). For this calculation we assumed $R_{b}=3 \sigma$.
To obtain such a prediction, we need to integrate the equation of motion. For molecules equipped with an EDM we start from Eq. (36). Using the definition of $T_{p}^{*}$ (33), this becomes

$$
\frac{d \vec{v} / \sigma}{d t}=\frac{1}{2} \frac{k_{b}}{M \sigma^{2}} T_{p}^{*}\left[2 \mathrm{n}\left(\frac{r^{2}}{\sigma^{2}}\right)-\frac{\sigma^{2}}{r^{2}} \mathrm{~N}\left(\frac{r^{2}}{\sigma^{2}}\right)\right] \frac{\vec{r} / \sigma}{r / \sigma} .
$$

Defining $\hat{r} \equiv \vec{r} / \sigma$, this transforms to

$$
\frac{d^{2} \hat{r}}{d t^{2}}=\frac{1}{2} \frac{k_{b} T_{p}^{*}}{M \sigma^{2}}\left[2 \mathrm{n}\left(\hat{r}^{2}\right)-\frac{1}{\hat{r}^{2}} \mathrm{~N}\left(\hat{r}^{2}\right)\right] \frac{\hat{r}}{|\hat{r}|} .
$$

Employing the rms transverse thermal velocity of the gas molecules,

$$
v_{\text {th }}=\sqrt{\frac{k_{b} T}{M}}
$$

we normalize the time coordinate as

$$
\hat{t}=t v_{\text {th }} / \sigma,
$$

and find

$$
\frac{d^{2} \hat{r}}{d \hat{t}^{2}} \frac{v_{\mathrm{th}}^{2}}{\sigma^{2}}=\frac{1}{2} \frac{k_{b} T_{p}^{*}}{M \sigma^{2}}\left[2 \mathrm{n}\left(\hat{r}^{2}\right)-\frac{1}{\hat{r}^{2}} \mathrm{~N}\left(\hat{r}^{2}\right)\right] \frac{\hat{r}}{|\hat{r}|} .
$$

Note that $v_{\text {th }} / \sigma$ is the rms inverse time a molecule would need to travel across the beam size $\sigma$.

Using the definition of the gas temperature, we obtain

$$
\frac{d^{2} \hat{r}}{d \hat{t}^{2}}=\frac{1}{2} \frac{T_{p}^{*}}{T}\left[2 \mathrm{n}\left(\hat{r}^{2}\right)-\frac{1}{\hat{r}^{2}} \mathrm{~N}\left(\hat{r}^{2}\right)\right] \frac{\hat{r}}{|\hat{r}|} .
$$

We observe that, in these dimensionless scaled coordinates, the velocity of the molecule is

$$
\frac{d \hat{r}}{d \hat{t}}=\frac{d \vec{r}}{d \hat{t}} \frac{1}{\sigma}=\vec{v} \frac{d t}{d \hat{t}} \frac{1}{\sigma}=\frac{\vec{v}}{v_{\text {th }}},
$$

which means that the initial distribution of the normalized velocities $d \hat{r} / d \hat{t}$ has a standard deviation 1 .

Recalling (39), the velocity $v_{\text {th }}$ and temperature are related via

$$
\begin{aligned}
\left(\frac{v_{\mathrm{th}}}{\sigma}\right)^{2} & =\frac{k_{b} T}{M \sigma^{2}}=\frac{k_{b} T_{p}^{*}}{M \sigma^{2}} \frac{T}{T_{p}^{*}} \\
& =\frac{p}{\pi \epsilon_{0} M c} \frac{I}{\sigma^{3}} \frac{T}{T_{p}^{*}}=\frac{20}{e} \omega^{2} \frac{T}{T_{p}^{*}} .
\end{aligned}
$$

Combining this with the definition of $v_{\text {th }}$, Eq. (55), and integrating the dynamics up to a certain time $t_{\max }$, the corresponding $\hat{t}_{\max }$ becomes 


$$
\hat{t}_{\max }=\sqrt{\frac{20}{e}} \sqrt{\frac{T}{T_{p}^{*}}} \omega t_{\max } .
$$

Note that the product $\omega t_{\max } /(2 \pi)$ is the number of oscillations $n_{e}$ performed by molecules near the equilibrium radius $r_{e}$ during the time interval $t_{\max }$. We now consider time intervals for which the number of oscillations around $r_{e}$ is constant, equal to $n_{e}$, so that $\omega t_{\max }=2 \pi n_{e}$. The corresponding $\hat{t}_{\max }$ is

$$
\hat{t}_{\max }=\sqrt{\frac{20}{e} \frac{T}{T_{p}^{*}}} 2 \pi n_{e} .
$$

This formula allows us to set $\hat{t}_{\max }$ as a function of $T / T_{p}^{*}$, so as to keep the same number of oscillations around the equilibrium radius $r_{e}$. In this way we can compare the molecular gas response to the beam for different temperatures $T$.

Figure 16 shows the simulated dynamics of few particles at zero temperature, $T=0$. In the left panel, the particles are launched only in the $x$ plane. The dynamics of the molecule pinch resembles the one of the electron-cloud pinch, e.g., the density evolution of cloud electrons during a bunch passage [14-16]. However, note that, in this case for the EDM, the particles oscillate linearly around the equilibrium radius $r_{e}=(\pi / 2) \sigma$, and not around $r=0$.

The right panel of Fig. 16 shows the same case of a cold molecule gas, but now distributed in the full circular beam pipe. The panel reveals a complex pattern, not easily interpreted.

A better way to visualize the process is to carry out simulations with many more particles and to compute the molecule density as a function of the radius. The result of such a simulation is shown in Fig. 17.

This simulation, along with all those that follow, considers an ensemble of $N_{\mathrm{mac}}=4 \times 10^{5}$ macroparticles. The dynamics is computed with a leapfrog scheme applied over 201 steps per oscillation length. At a given
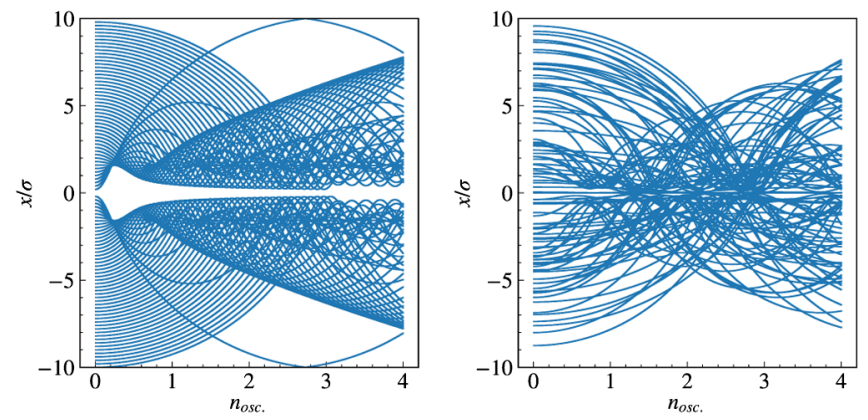

FIG. 16. Simulated pinch of the neutral EDM molecules in a cold gas, i.e., $T / T_{p}^{*}=0$. The particles are distributed either uniformly only in the $x$ plane (left) or randomly distributed (right) in a circular beam pipe of radius $R_{p}=10 \sigma$. integration step the local density $\rho(r)$ is obtained as $\rho(r)=\Delta N_{\text {mac }} /(2 \pi r \Delta r)$, with $\Delta N_{\text {mac }}$ denoting the number of macroparticles found in the radial shell $[r-\Delta r / 2$, $r+\Delta r / 2]$. The number of such shells in the interval $\left[0, r_{\max }\right]$ is 400 . Initially the molecules are distributed randomly and uniformly in the $(x / \sigma, y / \sigma)$ plane. The initial normalized velocities are chosen randomly according to a Gaussian distribution of standard deviation 1. For convenience, we normalize the particle density by division with the initial particle density $\rho_{0}$, computed as $\rho_{0}=N_{\text {mac }} /\left(\pi r_{\max }^{2}\right)$.

In the top panel of Fig. 17, we see the evolution of the local density normalized to the initial one. We observe a very pronounced pinch. The bottom panel instead displays
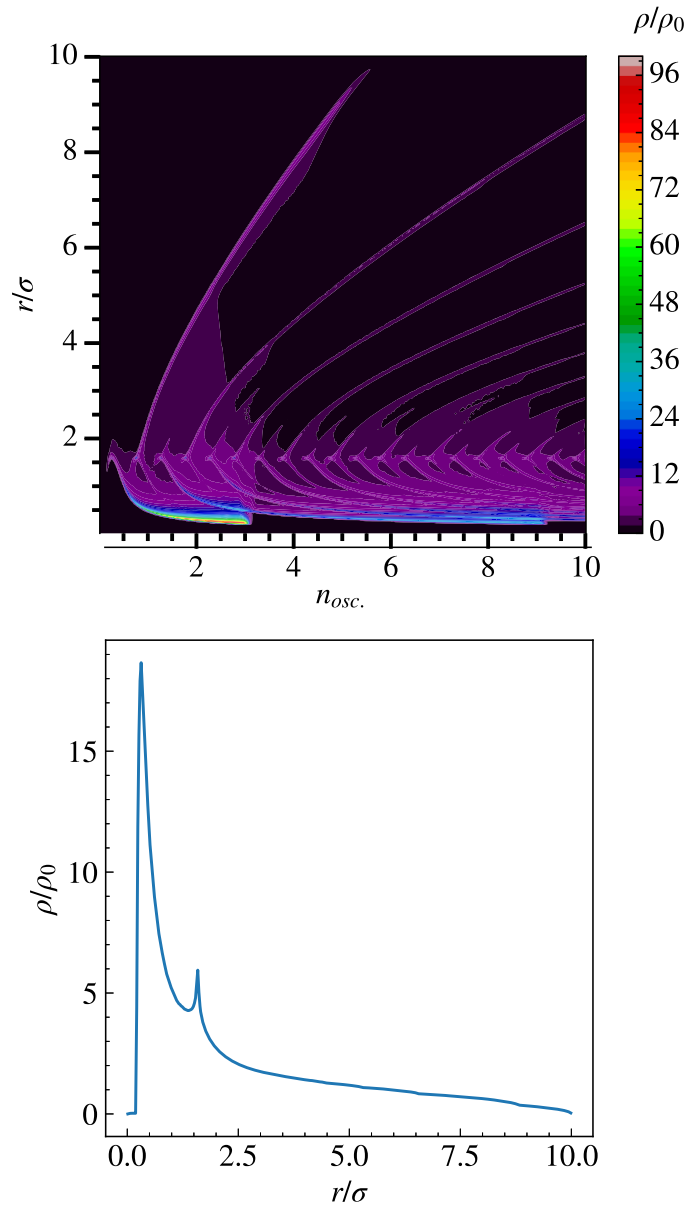

FIG. 17. EDM molecule density as a function of the radius and "time" (top) and the end time-averaged local molecule density versus radius (bottom). The molecule density is normalized with respect to the initial (or space-averaged) molecule density. The molecules are cold $T / T_{p}^{*}=10^{-5}$. In this simulation, the particles were distributed throughout the transverse $x-y$ space. At the bottom, the large peak of density is $\sim 20$ times the initial uniform distribution. Note the smaller peak of density, which reflects the local oscillations of molecules around the equilibrium radius $r / \sigma \simeq \pi / 2$; this phenomenon is clearly visible in the top panel as well. 

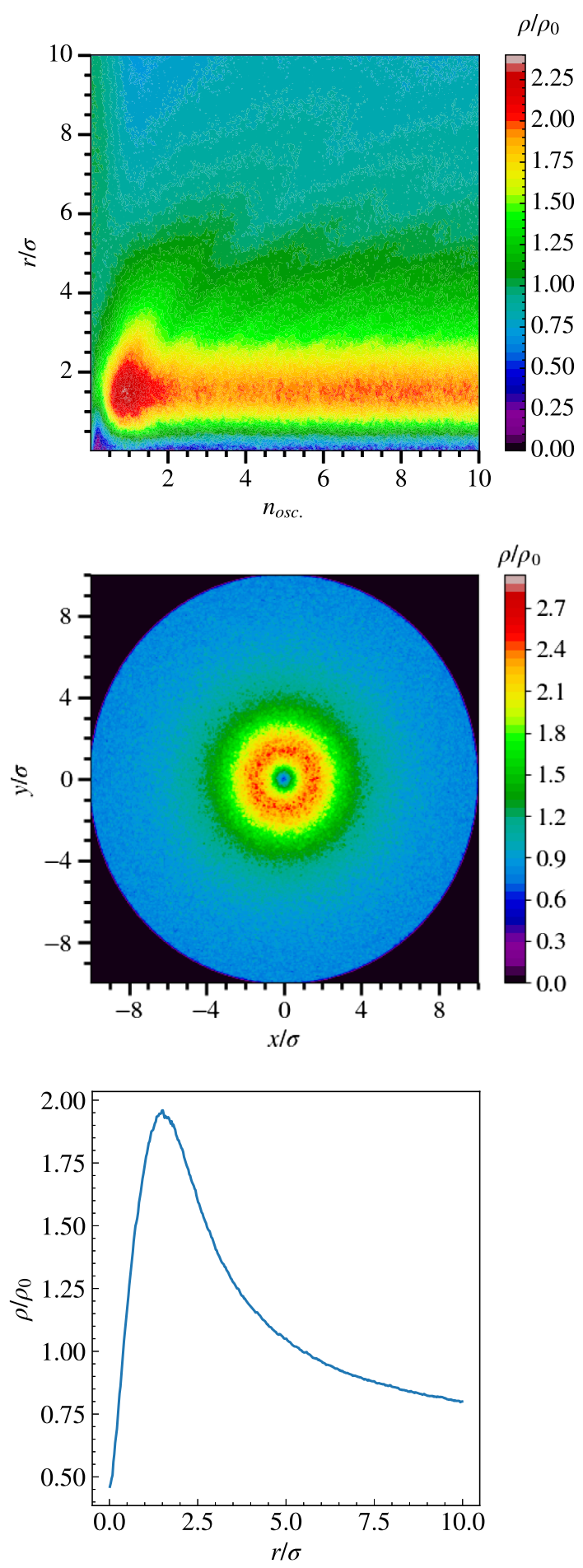

FIG. 18. Molecule density as a function of the radius and time (top), and the end time-averaged local molecule density versus radius (bottom). The center panel shows the $x-y$ structure of the molecule density at the point of maximum density. The molecule density is normalized with respect to the initial (or spaceaveraged) molecule density. In this simulation, the molecules are at a temperature $T / T_{p}^{*}=0.1$, and the particles initially distributed throughout the transverse $x-y$ space. According to the top panel (color scale), the peak density is $\sim 2.4$ times the initial uniform density.

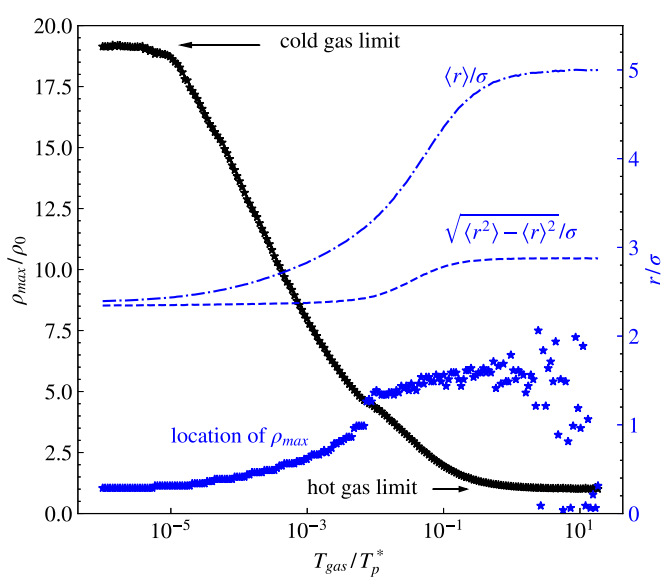

FIG. 19. Variation of the radial density distribution of EDM molecules with temperature $T / T_{p}^{*}$. Shown are the density enhancement due to the pinch in the beam field (black markers, left axis), the radial location of the maximum (blue markers, right axis), the average radial position of molecules (dash-dotted blue line), and the rms value of the radial position (dashed blue line).

the time-average density, normalized to the initial density $\rho_{0}$ (which always is equal to the average density), as a function of radial position $r$, revealing a peak average density close to the beam almost 20 times higher than the initial one. This simulation was performed at a temperature such that $T / T_{p}^{*}=10^{-5}$.

The density evolution strongly depends on the temperature, $T / T_{p}^{*}$. To illustrate this point, Fig. 18 shows the same set of pictures for a gas temperature $T / T_{p}^{*}=0.1$. We see that the nonzero temperature limits the effect of the pinch, and, in this case, we find a peak of only $\simeq 2.4 \rho_{0}$, i.e., two and a half times the initial density.

In view of the strong dependence of pinch density enhancement on $T / T_{p}^{*}$, we summarize the situation in a global picture presented in Fig. 19, where we have averaged over a time interval corresponding to ten oscillations near position $r_{e}$. The black markers indicate the normalized peak molecule density, with black values on the left vertical axis. For example, at $T / T_{p}^{*}=0.1$, we have $\rho_{\max } / \rho_{0} \simeq 1.9$, while for $T / T_{p}^{*}>1$ this ratio approaches $\rho_{\max } / \rho_{0} \simeq 1$ : To good approximation, for $T / T_{p}^{*}>5$ we find $\rho_{\max } / \rho_{0} \simeq 1$. The blue markers, with values on the right vertical axis, indicate the radial position at which the molecule density assumes its maximum value. We see that for $T / T_{p}^{*}>5$ the maximum molecule density spreads around $r_{e}=(\pi / 2) \sigma$. We also analyze the statistical properties of the distribution by computing the moments $\left\langle r^{n}\right\rangle=\int r^{n} P(r) d r$ using the probability density function $P(r)=c_{n} \rho(r)$, with $c_{n}$ a normalization constant ensuring that $\int P(r) d r=1$. The dash-dotted blue curve is the corresponding "average position" $\langle r\rangle$, computed as $\langle r\rangle=\int P(r) d r$ with the integral extending over the radial range from 0 to $10 \sigma$. For $T / T_{p}^{*}>5$ this curve is locked at a value of about 5 , 

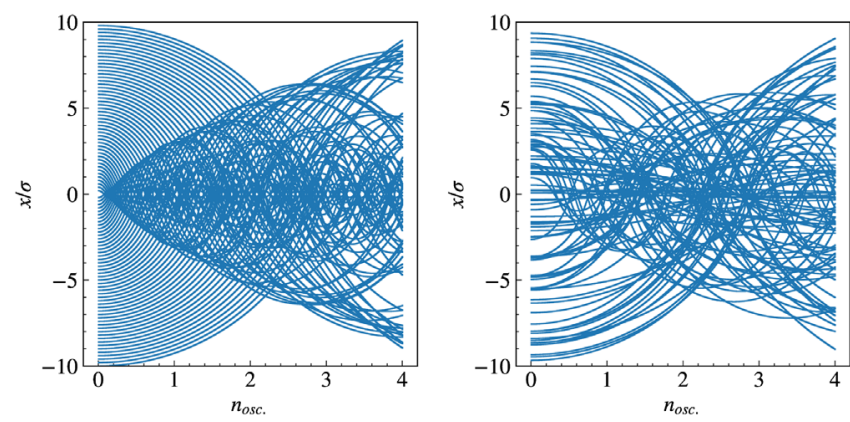

FIG. 20. Simulated pinch of the neutral MDM molecules in a cold gas, i.e., $T / T_{\mu}^{*}=0$. The particles are distributed either uniformly only in the $x$ plane (left) or randomly distributed (right) in a circular beam pipe of radius $R_{p}=10 \sigma$.
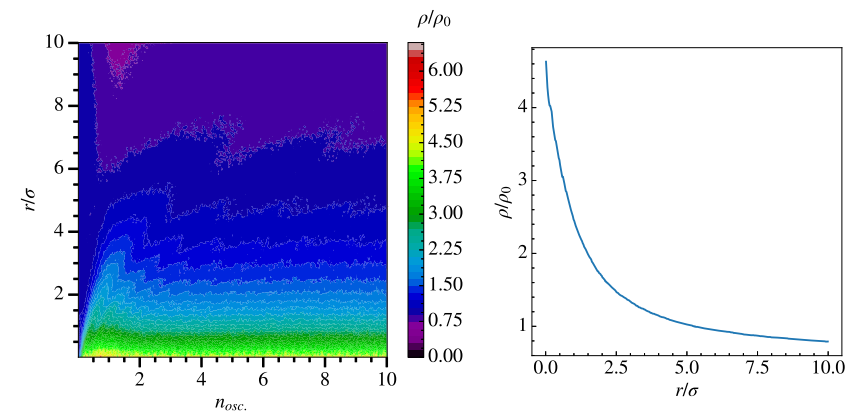

FIG. 21. MDM molecule density as a function of the radius and time (left) and the end time-averaged local molecule density versus radius (right). The molecule density is normalized with respect to the initial (or space-averaged) molecule density. In this simulation, the molecules are at a temperature $T / T_{\mu}^{*}=0.1$, and the particles initially distributed throughout the transverse $x-y$ space.

due to the fact that in this case the distribution $\rho(r)$ is uniform in $r$. For $T / T_{p}^{*}<3$, this curve tends towards the value 2.65 , reflecting the presence of a peak in the density.
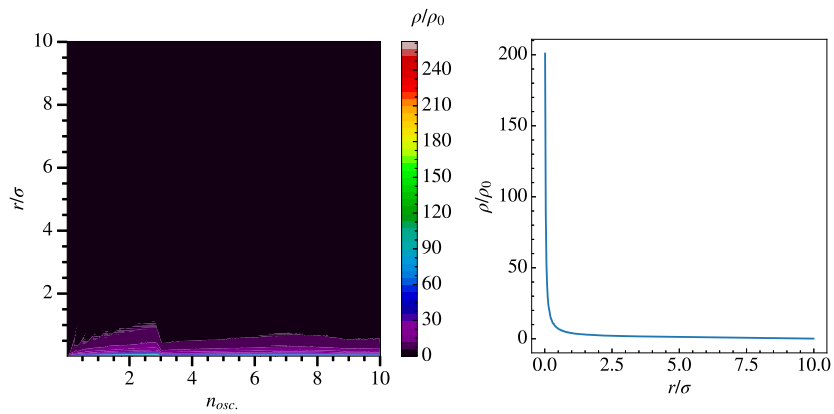

FIG. 22. MDM molecule density as a function of the radius and time (left) and the end time-averaged local molecule density versus radius (right). The molecule density is normalized with respect to the initial (or space-averaged) molecule density. In this simulation, the molecules are at a low temperature of $T / T_{\mu}^{*}=10^{-5}$, and the particles initially distributed throughout the transverse $x-y$ space.

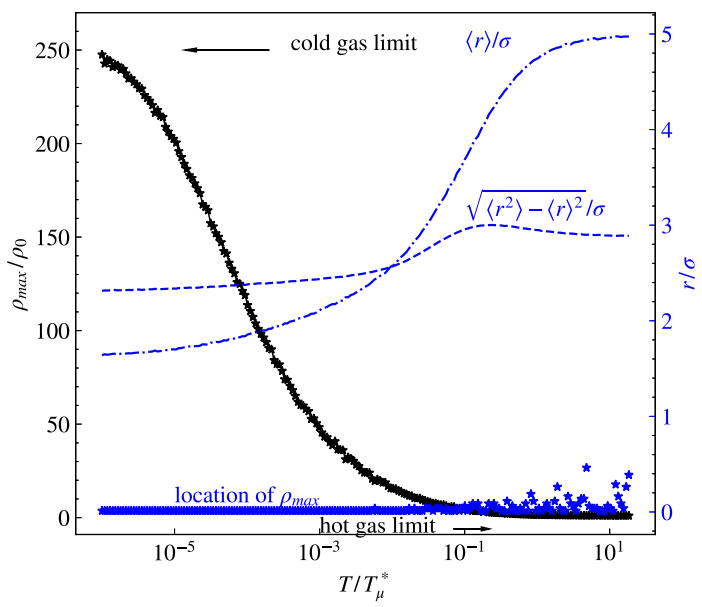

FIG. 23. Variation of the radial density distribution of MDM molecules with temperature $T / T_{\mu}^{*}$. Shown are the density enhancement due to the pinch in the beam field (black markers, left axis), the radial location of the maximum (blue markers, right axis), the average radial position of molecules (dash-dotted blue line), and the rms value of the radial position (dashed blue line).

The dashed blue curve shows the standard deviation of $r$, i.e., $\left(\left\langle r^{2}\right\rangle-\langle r\rangle^{2}\right)^{1 / 2}$, which indicates the width of the local peak. For a uniform distribution this value would be $10 / \sqrt{12}=2.88$, which indeed is reached at high temperatures in Fig. 19. For $T / T_{p}^{*}<3$ the standard deviation becomes smaller, assuming a value of $\sim 2.3$ for $T / T_{p}^{*}=0$, again signaling a nonuniformity in the distribution, namely the presence of a density peak, as is seen in Fig. 17.

Similar analyses can be carried out for molecules possessing a MDM and results analogous to those for the EDM are shown in Figs. 20-23. In Fig. 23 we observe that the cold gas limit for the MDM is an order of magnitude higher than for the EDM.

\section{DYNAMICS AND FORMATION OF FLAKES}

Inspired by our phenomenological discussion in Sec. IV, we now consider the possible presence of neutral flakes in the accelerator beam vacuum system.

The physical interactions between molecules which give rise to the formation of large and complex structures have long been the subject of extensive studies in the research field of "aggregation phenomena" [17,18]. Aggregation mechanisms are being investigated through particle-cluster and cluster-cluster models [19]. In particular, the dynamics of agglomeration for the case of particle-cluster models with dipolar interactions was studied in Refs. [20,21], which predicted the size and fractal dimension of the resulting clusters. Experiments and simulation models indicate that polar particles experience aggregations in which the original dipole moments are assembled into a cluster, whose specifics depend on the physical nature of the particles' dipole field. Studies of dust coagulation for 
particles carrying a MDM [22] revealed that the emerging cluster exhibits a total magnetic dipole moment which scales as $\mu \propto \mu_{0} N^{0.53}$, while for coagulating particles with an EDM the total dipole moment is only weakly dependent on $N$, in either case considering a plasma environment, not an accelerator-type vacuum. These studies also demonstrated that clustering starts when the velocity of the particles is sufficiently slow to permit "dipole-dipole trapping" [22].

We here suggest the possibility of agglomerate formation in particle accelerators, which would be driven by the history of an accelerator's beam vacuum system: sequences of events such as air leakage followed by cooling to ultralow temperature and subsequent intermediate warmup periods may foster the formation of aggregates, or flakes, in an ultrahigh vacuum at low temperature.

In the context of our discussion, we consider flakes of molecules as agglomerates composed of a large number $N$ of single molecules, each having a mass $M$ and EDM $p$ or MDM $\mu$. A flake may be held together by the forces between the (aligned) molecular dipole moments. A general flake thus has a mass $M_{f}=N M$, and a maximum electric dipole moment $p_{f}=N p$ or magnetic dipole moment $\mu_{f}=N \mu$. In either case, the initial condition is again determined by the thermal equilibrium temperature $T$, which remains the same as for the single molecules. The characteristic temperatures $T_{p}^{*}, T_{\mu}^{*}$ defined in Eqs. (33) and (34), respectively, are related to the corresponding trapping temperature of the flakes (suffix " $\mathrm{f}$ ") via

$$
T_{p, f}^{*}=N T_{p}^{*}, \quad T_{\mu, f}^{*}=N T_{\mu}^{*} .
$$

This reflects the fact that, at the same temperature, the flake has a much lower thermal velocity than a single molecule, and that it can more easily be trapped in the beam potential. Namely, if in Figs. 14 and 15 a single particle has a certain temperature $T / T_{p}^{*}$, the corresponding flake would have an $N$ times higher trapping temperature $T_{p, f}^{*}=N T_{p}^{*}$, or $T / T_{p, f}^{*}=\left(T / T_{p}^{*}\right) / N$. Hence, the point describing the flake lies much closer towards zero, on the left side of the diagrams, and, therefore, a significantly larger fraction of the flakes will be trapped, as indicated in the aforementioned panels.

As a concrete example, in the previously discussed case with molecules of $\mathrm{H}_{2} \mathrm{O}$, we found that $T_{p}^{*}=0.18 \mathrm{~K}$ and for this gas at $T=2 \mathrm{~K}$ we had $T / T_{p}^{*}=11.1$; in the case these molecules cluster to form a flake, for instance each containing $N=10,000$ molecules, we have $T_{p, f}^{*}=180 \mathrm{~K}$, so that at $T=2 \mathrm{~K}$ we find $T / T_{p, f}^{*}=0.0011$, and the majority of the flakes will be trapped.

More generally, in Figs. 19 and 23 it is quite evident that for $T / T_{p, f}^{*} \rightarrow 0$ or $T / T_{\mu, f}^{*} \rightarrow 0$, respectively, the maximum density of the pinched flakes becomes large, with potential consequences for the effective vacuum pressure and interaction with the beam. For the case of polar $\mathrm{H}_{2} \mathrm{O}$ flakes, the peak density in the pinch reaches about 20 times the initial value, whereas for paramagnetic $\mathrm{O}_{2}$ flakes the peak density even increases by a factor of 250. These estimates suggest that large polar flakes, clusters or dust particles in an accelerator along with the mechanisms of their formation deserve a more thorough investigation, as the process of dipolar assembly will lead to clusters subject to the flake dynamics presented, with a consequent risk of pinch and trapping in the beam field.

\section{CONCLUSIONS AND OUTLOOK}

Many neutral molecules possess a permanent electric or magnetic dipole moment. Their motion in an accelerator vacuum system will be perturbed by the electromagnetic field of the beam, leading to a possible trapping and density enhancement of such particles in the vicinity of the beam, especially in cold environments.

In this paper, we have analyzed the equations of motion of electrically or magnetically polar molecules, and identified the respective constants of motion. We derived the fraction of molecules, with either electric or magnetic dipole moment, trapped by the beam field, as a function of temperature, expressed in terms of a characteristic trapping temperature. The resulting local density enhancement was calculated as a function of radial position and time.

In particular, we have shown that molecules with a magnetic dipole moment oscillate around the transverse center of the particle beam, whereas molecules with an electric dipole moment oscillate around a radial equilibrium position located at the edge of the beam.

Observations of beam loss and beam instabilities in the 2017 and 2018 LHC runs cannot be explained by the motion of single neutral molecules, which, at a temperature of $5 \mathrm{~K}$, would mostly not be trapped by the field of the beam. However, the trapping of larger neutral flakes, or agglomerates of a large number of polar water or paramagnetic oxygen molecules, is indeed possible, and, if such flakes had been formed in the LHC, this could well have contributed to the magnitude of the observed phenomena. Such an explanation might also be consistent with the degraded situation encountered after a beam screen warm-up from about 5 to $80-90 \mathrm{~K}$ ("regeneration") around the LHC location 16L2 executed in August 2017 [5], since the higher temperature during the warm-up could have facilitated the formation of flakes.

Regarding the possible presence of flakes, the tools and methodologies developed for modeling aggregation phenomena [17-22] may serve as a starting point for future studies of cluster formation and flake characteristics in accelerator beam vacuum systems.

Throughout this article, we have analyzed the motion of uncharged objects carrying a dipole moment. Evidently, once a molecule or a flake comes close to the beam it may 
be ionized [23], from which point onward its dynamics is radically altered. For ionized molecules or ionized single atoms interacting with a charged particle beam the equations of motion are well known [24]. On the other hand, a larger flake staying near the core of the beam would heat up, be charged, and then either evaporate or melt and explode [25], leaving behind a localized high-density mixture of ions, electrons, and molecules or atoms. A software package is under development at CERN, to study the interaction of such a complex mixture of species with the LHC proton beam [26].

In general, the trapping and accumulation of individual neutral molecules or flakes of molecules in the vicinity of the beam enhances the effective gas density and can aggravate ion-induced beam instabilities, such as those discussed in Refs. [27-30]. The effect considered is particularly important in cryogenic vacuum systems, for high beam currents or for small beam sizes. Consequently, it will become more important for future generations of accelerators.

\section{ACKNOWLEDGMENTS}

This work was supported, in part, by the European Commission under the HORIZON2020 Integrating Activity project ARIES, Grant Agreement No. 730871. M. A. Rehman's research stay at CERN was supported by a grant from the Graduate University for Advanced Studies (SOKENDAI), Japan, by K. Furukawa of KEK, and by G. Arduini, M. Giovannozzi, and M. Vretenar of CERN. The authors also thank M. Bai and M. Steck of GSI, and U. Ratzinger of Goethe University Frankfurt for continuous encouragement.

\section{APPENDIX: ON THE ALIGNMENT CONDITION}

In this Appendix, we discuss the simplifying ansatz made throughout the main text regarding the alignment of the EDM $\vec{p}$ with $\vec{E}$, or of the MDM $\vec{\mu}$ with $\vec{B}$.

We consider a simple model of a molecule composed of two atoms: atom 1 with charge $+Q$ located at $\vec{r}_{1}$, and atom 2 with charge $-Q$ located at $\vec{r}_{2}$, each of the same mass $m$ (hence $M=2 m$ ), and kept apart at an equilibrium distance of $L$ meters, by a linear force. The distance $L$ is typically a small number. The center of mass of the molecule is $\vec{r}_{\mathrm{cm}}=\left(\vec{r}_{1}+\vec{r}_{2}\right) / 2$, and the location of atom 1 with respect the center of mass is $\vec{l}_{1}=\vec{r}_{1}-\vec{r}_{\mathrm{cm}}$, while for atom 2 the equivalent distance vector from the center is $\vec{l}_{2}=-\vec{l}_{1}$. Each atom is subject to a binding force that disappears when the atoms are separated by the equilibrium distance $L$. For atom 1 the binding force is

$$
\vec{N}_{1}=-k\left(\frac{1}{L}-\frac{1}{\left|\vec{r}_{1}-\vec{r}_{2}\right|}\right)\left(\vec{r}_{1}-\vec{r}_{2}\right),
$$

and for atom 2 it is $\vec{N}_{2}=-\vec{N}_{1}$. The constant $k$ characterizes the strength of the binding force keeping the two atoms together.

In addition, for each atom we also apply the external force $\vec{F}_{1}=\vec{F}_{2}=1 / 2 \vec{F}_{\text {cm }}$, with $\vec{F}_{\text {cm }}$ the force acting on the center of mass.

Finally, forces related to a torque $\vec{\tau}$ are assigned to the two atoms as

$$
\vec{F}_{1, \tau}=\frac{1}{2\left(l_{1}\right)^{2}} \vec{\tau} \times \vec{l}_{1}=\frac{1}{l^{2}} \vec{\tau} \times \vec{l},
$$

and $\vec{F}_{2, \tau}=-\vec{F}_{1, \tau}$, with $\vec{l}=\left(\vec{r}_{1}-\vec{r}_{2}\right)=2 \vec{l}_{1}$ and $l \equiv|\vec{l}|$.

The EDM is defined as $\vec{p}=Q \vec{l}$. The full set of equations necessary to characterize the motion of the molecule is

$$
\begin{aligned}
& \frac{d^{2} \vec{r}_{\mathrm{cm}}}{d t^{2}}=\frac{1}{2 m} \vec{F}_{\mathrm{cm}} \\
& \frac{d^{2} \vec{p}}{d t^{2}}=2 \frac{1}{m} \frac{Q^{2}}{p^{2}} \vec{\tau} \times \vec{p}-\frac{Q}{m} 2 k\left(\frac{1}{Q L}-\frac{1}{|\vec{p}|}\right) \vec{p} \\
& \vec{F}_{\mathrm{cm}}=(\vec{p} \cdot \nabla) \vec{E} \\
& \vec{\tau}=\vec{p} \times \vec{E} .
\end{aligned}
$$

Next, we define $p_{0}=Q L, \vec{p}_{n}=\vec{p} / p_{0}, p_{n}=\left|\vec{p}_{n}\right|$, and consider the electric field generated by a round beam as

$$
\vec{E}(r)=E_{0} A\left(\frac{r}{\sigma}\right) \frac{\vec{r}}{r}=E_{0} A\left(\frac{r}{\sigma}\right) \frac{\vec{r} / \sigma}{r / \sigma}=E_{0} \vec{E}_{n}\left(\frac{\vec{r}}{\sigma}\right)
$$

with $E_{0}$ the maximum electric field. For convenience we have defined $\vec{E}_{n}=\vec{E} / E_{0}$, which reads

$$
\vec{E}_{n}(\vec{v})=A(v) \frac{\vec{v}}{v}
$$

with, for a Gaussian beam,

$$
A(v)=\frac{1}{\ln (\pi / 2)} \frac{1}{v}\left(1-e^{-\frac{1}{2} v^{2}}\right) .
$$

The coefficient $\ln (\pi / 2)$ is a good approximation to the real normalization coefficient $\left(A_{\max }=0.99927\right.$ instead of $A_{\max }=1$ and is reached at $v=1.585201 \ldots$ approximately at $v=\pi / 2)$. We also define

$$
\omega_{E}^{2}=\frac{p_{0} E_{0}}{I_{i}}
$$

with $I_{i}=m L^{2} / 2$ the nominal momentum of inertia of the molecule. For a coasting beam the maximum electric field is 


$$
E_{0}=\ln \left(\frac{\pi}{2}\right) \frac{I}{2 \pi \epsilon_{0} c \sigma}
$$

with $I$ the beam current and $\sigma$ the transverse rms beam size.

By transforming the time $t$ to $\tau=t \omega_{E} /(2 \pi)$ and normalizing the position with the rms beam size $\sigma$, i.e., $\vec{r}_{n}=\vec{r}_{\mathrm{cm}} / \sigma$, the equations of motion become

$$
\begin{aligned}
\frac{d^{2} \vec{r}_{n}}{d \tau^{2}} & =\pi^{2} \frac{L^{2}}{\sigma^{2}}\left(\vec{p}_{n} \cdot \nabla_{r_{n}}\right) \vec{E}_{n}\left(\vec{r}_{n}\right) \\
\frac{d^{2} \vec{p}_{n}}{d \tau^{2}} & =(2 \pi)^{2}\left[\hat{p}_{n} \times \vec{E}_{n}\left(\vec{r}_{n}\right)\right] \times \hat{p}_{n} \\
& -(2 \pi)^{2} \frac{k}{Q E_{0}}\left(1-\frac{1}{p_{n}}\right) \vec{p}_{n} .
\end{aligned}
$$

A similar approach can be adopted for the equations of motion of binary molecules with a MDM. However, in this case we choose the molecule with $\vec{l}$ orthogonal to $\hat{z}$, and also $\vec{\mu}$ orthogonal to $\hat{z}$. We further define $\vec{l}_{n}=\vec{l} / L$. We can decompose $\vec{\mu}$ as $\vec{\mu}=|\mu|\left(\mu_{p} \hat{l}+\mu_{o} \hat{l} \times \hat{z}\right)$ with $\mu_{p}^{2}+\mu_{o}^{2}=1$.

In analogy to the EDM case, we introduce a normalized magnetic field,

$$
\vec{B}_{n}=A(v) \hat{r}_{n} \times \hat{z},
$$

so that $\vec{B}=B_{0} \vec{B}_{n}$, with $B_{0}$ designating the maximum magnetic field generated by the beam. Note that in this appendix the symbol $\hat{\nu}$ means $\hat{\nu}=\vec{\nu} /|\vec{\nu}|$. Setting

$$
\omega_{B}^{2}=\frac{|\mu| B_{0}}{I_{i}}
$$

we can write the equations of motion of the molecule as

$$
\begin{aligned}
\frac{d^{2} \vec{r}_{n}}{d \tau^{2}} & =\pi^{2} \frac{L^{2}}{\sigma^{2}}\left[\hat{\mu} \cdot \nabla_{n}\right] \vec{B}_{n} \\
\frac{d^{2} \vec{l}_{n}}{d \tau^{2}} & =(2 \pi)^{2} \frac{1}{l_{n}^{2}}\left[\hat{\mu} \times \vec{B}_{n}\right] \times \vec{l}_{n} \\
& -(2 \pi)^{2} \frac{k L}{|\mu| B_{0}}\left(1-\frac{1}{\left|\vec{l}_{n}\right|}\right) \vec{l}_{n} \\
\hat{\mu} & =\mu_{p} \hat{l}_{n}+\mu_{o} \hat{l}_{n} \times \hat{z},
\end{aligned}
$$

where now $\tau=t \omega_{B} /(2 \pi)$. We note that $\tau$ is a dimensionless variable, which counts the phase advance in units of $2 \pi$.

Following the same argumentation, as for the electric field, we estimate the maximum magnetic field for a coasting beam to be

$$
B_{0}=\ln \left(\frac{\pi}{2}\right) \frac{I \mu_{0}}{2 \pi \sigma} .
$$

From the above systems of equations, it is straightforward to show that if $\vec{p}_{n}$ is slightly tilted with respect to $\vec{E}_{n}$, it oscillates around $\vec{E}_{n}$ at a frequency $\omega_{E}$. The identical behavior occurs for $\vec{\mu}$, which oscillates at a frequency $\omega_{B}$ around $\vec{B}_{n}$.

The two equations, (A5) and (A8), also reveal that the acceleration of the center of mass $\vec{r}_{n}$ is proportional to $\pi^{2} L^{2} / \sigma^{2}$. This means that the motion of the center of mass is slow. During one oscillation period of the EDM or MDM, the center of mass practically does not move. We also see that the binding force enters into the dynamics. However, we assume that this internal restoring force is much bigger than the additional forces exerted by the external beam magnetic or electric fields. This assumption implies that, to good approximation, $\left|\vec{l}_{n}\right|$ is constant, equal to 1. Expanding the force on the center of mass acting on a molecule with MDM we find

$$
\vec{F}=(\vec{\mu} \cdot \nabla) \vec{B}=[\vec{\mu} \cdot \hat{r}] B^{\prime}(r) \hat{B}-[\vec{\mu} \cdot \hat{B}] \frac{B(r)}{r} \hat{r} .
$$

If instead the molecule has an EDM we find the analogous equation

$$
\vec{F}=(\vec{p} \cdot \nabla) \vec{E}=[\vec{p} \cdot \hat{r}] E^{\prime}(r) \hat{r}+[\vec{p} \cdot \hat{B}] \frac{E(r)}{r} \hat{B},
$$

where $\hat{r}=\vec{r} / r, \hat{B}=\vec{r} / r \times \hat{z}$ and $\vec{p}=p_{x} \hat{x}+p_{y} \hat{y}$ and $\vec{\mu}=\mu_{x} \hat{x}+\mu_{y} \hat{y}$ and the electric and magnetic fields $\vec{E}(\vec{r})=E(r) \hat{r}, \vec{B}(\vec{r})=B(r) \hat{B}$. During the time of one oscillation of $\vec{\mu}$ or $\vec{p}$ around their equilibrium position, the maximum displacement of the center of mass is proportional to the ratio $\pi^{2} L^{2} / \sigma^{2}$ which is immensely small, typically $L=10^{-10} \mathrm{~m}$, and $\sigma=10^{-3} \mathrm{~m}$, hence $\pi^{2} L^{2} / \sigma^{2} \sim 10^{-13}$.

Therefore, we can look at the system on a timescale $T$ during which many oscillations of $\vec{\mu}_{n}$ or $\vec{p}_{n}$ around their equilibrium position occur, while the center of mass still moves little. This, in turn, implies that the quantities $B(r)$, $E(r)$, and $r$ are practically constant over the interval of time $T$. Hence, defining $\langle\cdot\rangle$ as an average over the time interval $T$, we find

$$
\langle\vec{F}\rangle=\langle\vec{\mu} \cdot \hat{r}\rangle B^{\prime}(r) \hat{B}-\langle\vec{\mu} \cdot \hat{B}\rangle \frac{B(r)}{r} \hat{r}
$$

for the MDM and

$$
\langle\vec{F}\rangle=\langle\vec{p} \cdot \hat{r}\rangle E^{\prime}(r) \hat{r}+\langle\vec{p} \cdot \hat{B}\rangle \frac{E(r)}{r} \hat{B}
$$

for the EDM. However, because the number of oscillations of $\vec{\mu}, \vec{p}$ is huge on the timescale $T$, we can simplify some terms as $\langle\vec{\mu} \cdot \hat{r}\rangle=0,\langle\vec{\mu} \cdot \hat{B}\rangle=\mu_{\text {eff }}$ and $\langle\vec{p} \cdot \hat{r}\rangle=p_{\text {eff }}$, $\langle\vec{p} \cdot \hat{B}\rangle=0$, and we only remain with

$$
\langle\vec{F}\rangle=-\mu_{\mathrm{eff}} \frac{B(r)}{r} \hat{r}
$$


and

$$
\langle\vec{F}\rangle=p_{\mathrm{eff}} E^{\prime}(r) \hat{r} .
$$

In other words, we can replace the force acting on the center of mass by an effective force resulting from an effective EDM, and an effective MDM aligned with the respective fields. By making this approximation we give up any attempt at resolving the tiny fluctuations of the center of mass deriving from the coupling with the fast oscillations of the MDM, or EDM, in the plane orthogonal to the field lines.

In addition, we note that the electric or magnetic field generated by the stored particle beam never appears suddenly, but rather is turned on slowly as the beam particles are injected or accumulated. This means that the fields $\vec{E}$ and $\vec{B}$ appear mostly adiabatically. This additional effect further fosters the alignment of the EDM and MDM to their respective field. We show this by a simulation, which illustrates that $\vec{\mu}$, and $\vec{p}$ spontaneously align with the local magnetic or electric field, respectively. Unfortunately, simulations for the real parameters are beyond computational reach, as they would require resolving the vibrational motion and the center of mass dynamics for $\pi^{2} L^{2} / \sigma^{2} \sim 10^{-13}$. Instead, we test the model dynamics for the previously described bi-atomic model with the "relaxed simulation parameters" of $L^{2} / \sigma^{2}=10^{-4}$ and $k /\left(Q E_{0}\right)=10^{3}$.

The two top panels in Fig. 24 compare the angle of the electric field at the location of the molecule (red) and the angle of the electric dipole moment $\vec{p}$ (blue), both with respect to the horizontal direction, on two different timescales. Figure 24 top left shows, in blue color, the angle of the electric dipole moment. For this simulation, the initial angle of $\vec{p}$ was chosen at $180^{\circ}$ with respect to $\vec{E}_{n}$, and the molecule was launched with zero rotational energy. This is the worst possible scenario. The picture reveals an oscillation of $\vec{p}$, whose angle more and more closely approaches the angle of the electric field. The center-left panel presents the situation at the end of the adiabatic turn-on, clearly demonstrating an alignment of $\vec{p}$ with $\vec{E}_{n}$. Note that, although the length of the time interval is the same as for the top panel, the red curve (the electrical field at the location of the molecule) now exhibits a larger change of angle. This feature stems from the dynamics experienced by the molecule's center of mass. At the end of the adiabatic ramping, the gradient of $E(r)$ is much larger than at the start of the field ramp, leading to a faster motion of the molecule around and across the beam. Consequently the red curve appears with a more pronounced slope, and the thickness of the blue curve reflects an extremely rapid oscillation of the residual orthogonal component of the dipole moment around the electric field line. Exactly the same reasoning applies to the example of a molecule equipped with a magnetic dipole moment and the simulation results for such
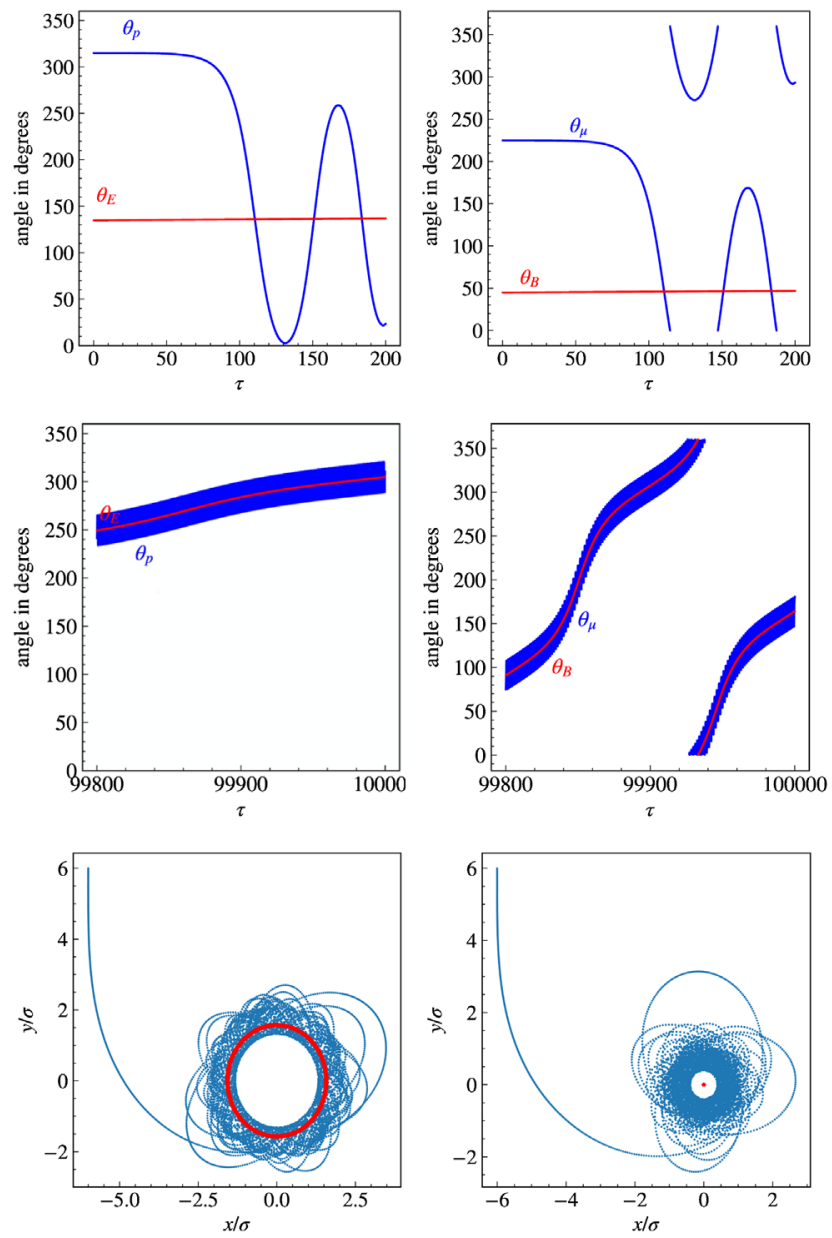

FIG. 24. Simulated angles of a molecule's $\vec{p}$ (blue) and local electric field $\vec{E}$ (red), with respect to the horizontal direction, at the start (top left) and end (center left) of an adiabatic increase of the electric field of the beam, over a total time $T$ that would correspond to $\tau=10^{5}$ periods of phase advance $\omega_{E} T$. The panels on the right present the simulated angular direction of a molecule's magnetic dipole moment $\vec{\mu}$ (blue) and of the local magnetic field $\vec{B}$ (red) during an analogous ramp-up of the beam's magnetic field, over the same number of periods of phase advance $\omega_{B} T$. The top and center panels each present the behavior over a time interval $\Delta \tau=200$. The bottom panels show the molecules' trajectories over the full simulation. The red circle in the bottom left panel indicates the equilibrium position $r_{e}$-compare Fig. 13 and Eq. (35).

a case are quite similar, as is shown in the three pictures on the right-hand side of Fig. 24. Also here a nearly perfect alignment is reached at the end of the adiabatic ramp-up of the magnetic field.

The simulations of Fig. 24 consider a slow turn-on of the electric field over a time interval $T$ corresponding to $\tau_{\max }=10^{5}$, or to the equivalent of $10^{5}$ oscillation periods for the maximum field at the end of the adiabatic ramp, where $\left|\vec{E}_{n}\right|=1$. Note that although this number of oscillations seems large, it does correspond to a rather short actual time interval, typically below a microsecond. 
Using Eq. (A4), which yields the maximum field for a Gaussian beam, and the definition of $T_{p}^{*}$ we find for a coasting beam

$$
\omega_{E}^{2} \simeq \ln \left(\frac{\pi}{2}\right) \frac{k_{b}}{m L^{2}} T_{p}^{*}
$$

Then, as an example, considering $\mathrm{H}_{2} \mathrm{O}$ and $T_{p}^{*}=0.18 \mathrm{~K}$, and by choosing the $L$ so as to obtain the correct moment of inertia for the equivalent bi-atomic model, namely $L=$ $0.65 \AA$ we find $\omega_{E}=1.33 \times 10^{11} \mathrm{rad} / \mathrm{s}$; one oscillation period is $\delta t=4.7 \times 10^{-11} \mathrm{~s}$. Therefore, in this example $10^{5}$ oscillations correspond to $\Delta t=4.7 \times 10^{-6} \mathrm{~s}$, and we have shown this to be sufficiently adiabatic for ensuring the alignment of EDM with $\vec{E}$, in the case of the relaxed simulation parameters. Note that even with a 10 times faster ramp as in $\Delta t=4.7 \times 10^{-7} \mathrm{~s}$, the alignment would still be reached to a good approximation. This timescale is comparable to the bunch spacing in the LHC.

The analysis for the MDM molecules can follow the same line of thought. Considering the maximum $B$ field given by Eq. (A9), and using the definition of $T_{\mu}^{*}$, we find

$$
\omega_{B}^{2} \simeq \ln \left(\frac{\pi}{2}\right) \frac{k_{b}}{m L^{2}} T_{\mu}^{*} .
$$

As an example, for $\mathrm{O}_{2}$ we had $T_{\mu}^{*}=2.5 \mathrm{mK}$; hence $\omega_{B}=$ $6.34 \times 10^{9} \mathrm{rad} / \mathrm{sec}$ and one oscillation period at the peak field is $\delta t=9.91 \times 10^{-10} \mathrm{~s}$. The duration of an adiabatic ramp over $10^{5}$ such oscillation periods corresponds to $\Delta t=9.91 \times 10^{-5} \mathrm{~s}$. This suggests a stiffer behavior of the MDM, compared with the EDM. In consequence, the EDM motion may not resolve individual bunch passages, but will align to a mean $B$ field.

We can analyze the process of alignment by using the concept of adiabatic invariants. In fact, the MDM and EDM oscillate around their respective equilibrium direction with angular frequencies $\omega_{E}$ or $\omega_{B}$, defined in Eqs. (A3) and (A7). For the orthogonal components of the dipole moments, the corresponding equations of motion are

$$
\frac{d^{2} p_{\perp}}{d t^{2}}+\omega_{E}^{2} p_{\perp}=0, \quad \frac{d^{2} \mu_{\perp}}{d t^{2}}+\omega_{B}^{2} \mu_{\perp}=0 .
$$

After transforming (A11) to the scaled time coordinate $\tau$, and, for convenience using the normalized variables $\vec{p}_{n}=\vec{p} /|\vec{p}|, \vec{\mu}_{n}=\vec{\mu} /|\vec{\mu}|$, the equivalent set of equations reads

$\frac{d^{2} p_{n, \perp}}{d \tau^{2}}+\Omega_{E}^{2} p_{n, \perp}=0, \quad \frac{d^{2} \mu_{n, \perp}}{d \tau^{2}}+\Omega_{B}^{2} \mu_{n, \perp}=0$,

where $\Omega_{E}$ and $\Omega_{B}$ vary with the normalized time $\tau$ and at maximum field (in our examples typically reached at $\left.\tau=\tau_{\max }\right)$ assume the value $2 \pi$.
Therefore, for small oscillation amplitudes, the dimensionless scaled time $\tau$ indicates how many oscillations the EDM, or MDM, would perform around the equilibrium direction if the field were maximum, i.e., for $E=E_{0}$ or $B=B_{0}$.

For example, taking the example of the electric field $E$, the adiabatic condition is

$$
\frac{|\vec{E}|}{|\dot{\vec{E}}|} \gg \frac{2 \pi}{\omega_{E}},
$$

where $\dot{E}$ is the time derivative. Considering an electric field that linearly rises from 0 to a value $E_{0}$ during the time interval $t_{\max }$, the adiabatic condition is fulfilled if $t^{3 / 2} \omega_{E_{0}} /(2 \pi) \gg t_{\max }^{1 / 2}$. This inequality constrains the time $t$ at which the field change becomes adiabatic.

An adiabatically rising $\vec{E}_{n}$ or $\vec{B}_{n}$ slowly changes the angular frequencies $\omega_{E}$ and $\omega_{B}$ until they approach their final values. In the new coordinate frame, the slowly varying $\Omega_{E}$ or $\Omega_{B}$ will grow from an initial value of zero to adiabatically reach the final value $\Omega_{B}=2 \pi$ or $\Omega_{E}=2 \pi$ at $\tau=\tau_{\max }$.

We have introduced the symbols $p_{n, \perp}$ and $\mu_{n, \perp}$, which denote the projections of $\vec{p}_{n}, \vec{\mu}_{n}$ onto the transverse axis perpendicular to $\vec{E}$ or $\vec{B}$, respectively. In the orthogonal dipole moment "phase space" $\left(p_{n, \perp}, d p_{n, \perp} / d t\right)$ or $\left(\mu_{n, \perp}, d \mu_{n, \perp} / d t\right)$, we can recognize the key features of adiabatic invariance. For the case of an EDM molecule, the top-left panel of Fig. 25 presents the dipole-moment evolution in the perpendicular "normalized" phase space obtained by using the time coordinate $\tau$. The model parameters for this simulation are the same as those for Fig. 24. The picture clearly reveals that the trace of the phase-space coordinate $\left(p_{n, \perp}, d p_{n, \perp} / d \tau\right)$ converges towards an ellipse. Based on the same set of simulation data, the top-right panel of Fig. 25 illustrates the evolution of the normalized dipole-moment total energy

$$
\frac{\mathcal{E}_{\perp}}{\Omega_{E}}=\frac{1}{\Omega_{E}}\left[\frac{1}{2}\left(\frac{d p_{n, \perp}}{d \tau}\right)^{2}+\frac{1}{2} \Omega_{E}^{2} p_{n, \perp}^{2}\right] .
$$

This picture confirms that, to good approximation, $A_{\text {inv }} \equiv \mathcal{E}_{\perp} / \Omega_{E} \simeq$ constant, once the field change has become adiabatic.

The actual value of $A_{\text {inv }}$ is determined by the initial conditions of the particle's dipole moment considered, i.e., by the initial value of $\vec{r}_{n}, d \vec{r}_{n} / d \tau, \vec{p}_{n}, d \vec{p}_{n} / d \tau$, and by the initial speed of turning on the electric or magnetic field, proportional to $1 / \tau_{\max }$. The field turn-on corresponds to a physical ramp time of

$$
T_{\text {ramp }}=\frac{2 \pi}{\omega_{E}} \tau_{\max },
$$


which unveils the equivalence of the dynamics for corresponding pairs of $\left(T_{\text {ramp }}, E_{0}\right)$. The dependence of $A_{\text {inv }}$ on $\tau_{\max }$ is examined in Fig. 25 (bottom left) for a linear ramp considering a particle with the same initial condition (in the $\tau$ frame). The bottom-right panel of Fig. 25 illustrates the dependence of $A_{\text {inv }}$ on $\tau_{\max }$ for a Gaussian ramp starting at a time of $5 \sigma_{z} / c$ before the arrival of the bunch center, where $\sigma_{z}$ denotes the rms bunch length. Comparing the evolution
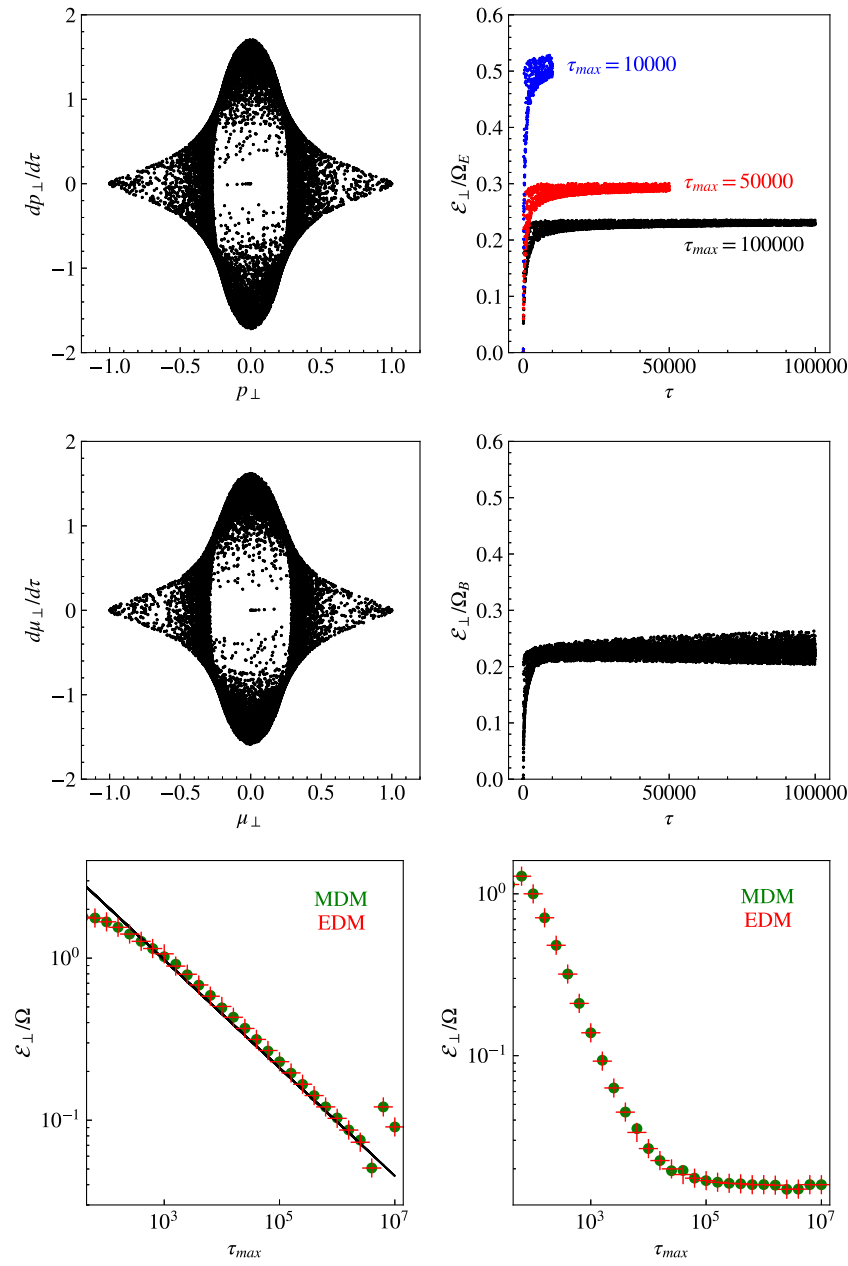

FIG. 25. Electric dipole moment phase space orthogonal to the local field direction, $\left(p_{\perp}, d p_{\perp} / d \tau\right)$ (top left), and the adiabatic invariant $A_{\text {inv }}$ as a function of the number of oscillations (top right). The right panel presents data for three different values of $\tau_{\max }$. The middle two panels present analogous simulation results for the case of the magnetic dipole moment. At the beginning of each simulation, large oscillations of $p_{\perp}$ or $\mu_{\perp}$ are seen, which later on converge onto an ellipse. The width of this ellipse in the direction of $p_{\perp}$, or $\mu_{\perp}$, becomes smaller the larger $\tau_{\max }$ is. Recalling Eq. (A15), $\tau_{\max }$ can be expressed as $\tau_{\max }=T_{\text {ramp }} /(2 \pi)\left(|p| E_{0} / I_{i}\right)^{1 / 2}$, and, therefore, the width of the ellipse in the direction of $p_{\perp}$ becomes smaller the more adiabatically the field changes (larger $T_{\text {ramp }}$ ) and the higher the final field is (higher $E_{0}$ ). This is demonstrated in the bottom panel by the dependence of $A_{\text {inv }}$ on $\tau_{\text {max }}$, both for a linear (left) and for a Gaussian ramp (right). for several different $\tau_{\max }$, the top-right panel of Fig. 25 shows that the value of $A_{\text {inv }}$ decreases as $\tau_{\max }$ increases. These findings reveal that $\tau_{\max }$ directly relates to the degree of alignment with respect to the field. In fact, the maximum excursion of $p_{n, \perp}$ at the end of the ramp, which we designate as $p_{n, \perp, \max }$, is given by

$$
p_{n, \perp, \max }=\sqrt{\frac{A_{\text {inv }}\left(\tau_{\max }\right)}{\pi}} .
$$

Therefore, if $A_{\text {inv }}$ shrinks with increasing $\tau_{\max }$, so does $p_{n, \perp, \max }$.

To shed some light on the dependence of $A_{\text {inv }}$ on $\tau_{\max }$, we again consider the case of a linear ramp, where the electric field increases linearly in time, and the frequency $\Omega_{E}$ evolves as

$$
\Omega(\tau)=2 \pi \sqrt{\frac{\tau}{\tau_{\max }}}
$$

At the beginning of the ramp, the fields are too weak to meet the adiabatic condition, (A13), which in these coordinates assumes the particularly simple form

$$
\tau \gg \tau_{\max }^{1 / 3}
$$

This condition will start to hold true after a time $\bar{\tau}$ large enough to allow for a significant phase advance of the oscillation of the perpendicular dipole-moment component around the electric or magnetic field. The phase advance of this oscillation is estimated as

$$
\Phi(\tau)=\int_{0}^{\tau} \Omega_{E}(x) d x=2 \pi \frac{2}{3} \frac{\tau^{3 / 2}}{\sqrt{\tau_{\max }}} .
$$

We denote by $f$ the phase advance in unit of $2 \pi$ required to enter into the adiabatic domain, so that the adiabatic process will start when

$$
\Phi(\bar{\tau})=2 \pi f,
$$

that is, by the time

$$
\bar{\tau}=f^{2 / 3}\left(\frac{3}{2}\right)^{2 / 3} \tau_{\max }^{1 / 3} .
$$

At this moment the corresponding $\Omega_{E}$ is

$$
\Omega_{E}(\bar{\tau})=2 \pi \sqrt{\frac{\bar{\tau}}{\tau_{\max }}}=2 \pi f^{1 / 3}\left(\frac{3}{2}\right)^{1 / 3} \tau_{\max }^{-1 / 3} .
$$

This corresponds to the value of $\Omega$ which sets the value of $A_{\text {inv. }}$. In fact, from 


$$
A_{\mathrm{inv}}=\frac{1}{\Omega_{E}}\left[\frac{1}{2}\left(\frac{d p_{n, \perp}}{d \tau}\right)^{2}+\frac{1}{2} \Omega_{E}^{2} p_{\perp}^{2}\right],
$$

and neglecting the kinetic term, which is small for our test particle (defined below), we find

$$
A_{\text {inv }}=\frac{1}{2} \Omega_{E}(\bar{\tau}) p_{\perp}^{2} .
$$

We note that our test particle is launched at a distance far from the beam center and with a velocity sufficiently low such that at the time by which the phase advance becomes $2 \pi f$, i.e., by when the adiabatic condition is met, the particle still remains far away, with $p_{\perp}$ unchanged.

Substituting for $\Omega_{E}$ the value at which the adiabatic process starts, we obtain

$$
A_{\text {inv }}=\pi f^{1 / 3} p_{\perp}^{2}\left(\frac{3}{2}\right)^{1 / 3} \tau_{\max }^{-1 / 3} .
$$

Our simulation results are shown by the markers in Fig. 25 (bottom left). Although the previous model is valid only for small oscillations around the equilibrium angle of the dipole moment, we find that choosing the values $f=20$ and $p_{\perp}=1$, Eq. (A22) fits these simulation results extremely well.

Note that this discussion, including the results shown in Fig. 25, is carried out in the reference system with normalized time $\tau$. This implies that the initial condition in physical coordinates, $d \vec{r}_{n} / d t$, is related to the initial condition in normalized space, $d \vec{r}_{n} / d \tau$, according to

$$
\frac{d \vec{r}_{n}}{d t}=\frac{d \vec{r}_{n}}{d \tau} \frac{d \tau}{d t}=\frac{d \vec{r}_{n}}{d \tau} \frac{\omega_{E}}{2 \pi} .
$$

The initial rms velocity of a molecule follows from the thermal equilibrium with the environment as $v_{\text {th }}=$ $\sqrt{k_{b} T / M}$, and $\omega_{E}$ is given by Eq. (A10). Therefore, Eq. (A23) becomes

$$
\left(\frac{d r_{n}}{d \tau}\right)_{\mathrm{rms}}=\frac{2 \pi}{\sqrt{\ln (\pi / 2)}} \frac{L}{\sigma} \sqrt{\frac{m}{M} \frac{T}{T_{p}^{*}}} .
$$

As $L$ is of the order of magnitude $\sim 10^{-10} \mathrm{~m}$ and $\sigma \sim 10^{-3} \mathrm{~m}$, and as $m / M \leq 1$ is always fulfilled, even for $T / T_{p}^{*}=10^{6}$ we would find $\left(d r_{n} / d \tau\right)_{\mathrm{rms}} \sim 6 \times 10^{-4}$. Hence, we may consider the initial condition of molecules in the system of coordinates $\tau$ as effectively frozen. The same argument applies to the MDM case, after replacing $T_{p}^{*}$ with $T_{\mu}^{*}$.

It follows that when the electric field rises adiabatically, the oscillation amplitude of $p_{\perp}$ slowly decreases, so that $\vec{p}$ more and more aligns with $\vec{E}$, independently of the motion of the center of mass. The same argument applies to the
MDM case as is illustrated in the center panels and by the green markers in the bottom panels of Fig. 25.

The previous analysis is now discussed for bunched beams. Considering the passage of a single round bunch, the maximum electric field is obtained from Eq. (A4) with $I$ now the maximum current at the bunch center and $\sigma$ the transverse rms beam size. For a Gaussian bunch profile, the longitudinal charge distribution is

$$
\rho_{z}(z)=\frac{q N_{b}}{\sqrt{2 \pi} \sigma_{z}} e^{-\frac{1 z^{2}}{2 \sigma_{z}^{2}}}
$$

with $N_{b}$ the number of particles in the bunch, $q$ the charge per beam particle, and $\sigma_{z}$ the rms bunch length. Hence, for this case the peak current reads $I=c q N_{b} /\left(\sqrt{2 \pi} \sigma_{z}\right)$, and, finally, we can express the maximum electric field as

$$
E_{0}=\frac{\ln (\pi / 2)}{(2 \pi)^{3 / 2}} \frac{q N_{b}}{\epsilon_{0} \sigma \sigma_{z}} .
$$

For LHC bunches at top energy with $\sigma=2 \times 10^{-4} \mathrm{~m}$, $\sigma_{z}=0.076 \mathrm{~m}, N_{b}=1.2 \times 10^{11}$ protons, we obtain a maximum electric field of $E_{0} \approx 4 \times 10^{6} \mathrm{~V} / \mathrm{m}$.

Following the same argumentation, as for the electric field, we estimate the maximum magnetic field during a bunch passage from Eq. (A9), and using the maximum beam current, we now find

$$
B_{0}=\frac{\ln (\pi / 2)}{(2 \pi)^{3 / 2}} \frac{\mu_{0} c q N_{b}}{\sigma \sigma_{z}} .
$$

Inserting the same reference LHC beam parameters as before yields the estimate $B_{0}=137 \times 10^{-4} \mathrm{~T}=137$ Gauss.

Considering the LHC bunches, for $\mathrm{H}_{2} \mathrm{O}$ molecules Eq. (A3) yields a maximum angular oscillation frequency $\omega_{E} \approx 9.0 \times 10^{11} \mathrm{rad} / \mathrm{s}$, corresponding to $T_{p}^{*} \approx 8.1 \mathrm{~K}$, and, with a ramp time $T_{\text {ramp }}=5 \sigma_{z} / c$, Eq. (A15) yields $\tau_{\max } \approx 181$. On the other hand, for an $\mathrm{O}_{2}$ molecule, the peak angular oscillation frequency is $\omega_{B}=4.27 \times 10^{10} \mathrm{rad} / \mathrm{s}$, corresponding to $T_{\mu}^{*}=0.11 \mathrm{~K}$ and $\tau_{\max } \simeq 8$.6. Inspecting Fig. 25 , for example, at $\tau_{\max } \simeq 181$ we find $A_{\text {inv }} \simeq 0.5$, and, for the perpendicular dipole moment at the end of the field ramp, i.e., at the passage of the bunch center, this implies $p_{n, \perp \text { max }} \simeq 0.4$, which corresponds to a residual misalignment of $\Delta \theta \simeq 23.5$ degree.

These numbers suggest that for the short bunches of the LHC the alignment condition is not strictly fulfilled and that, therefore, a residual oscillation of the dipole moments may affect the dynamics. However, the simulations presented in this Appendix were performed primarily in order to confirm that a process of alignment takes place at all, and these numerical studies were carried out for unrealistically "relaxed" model parameters, which allowed 


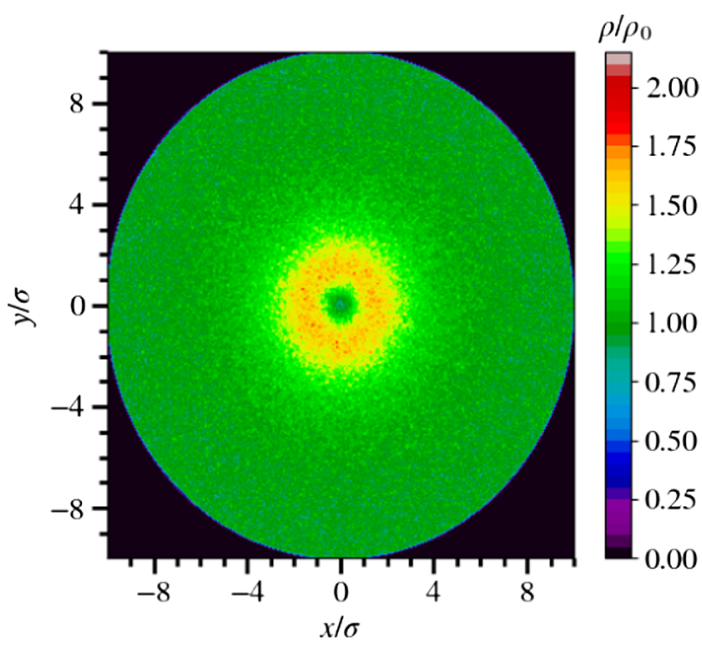

FIG. 26. Simulated density based on a rigid-molecule model, for a temperature $T / T_{p}^{*}=0.1$, at the normalized time $n_{\text {osc. }}=1$, which is approximately the moment of maximum peak density. The picture presents a snapshot of the molecule distribution in the $x-y$ plane. The molecule density is normalized with respect to the initial (or space-averaged) molecule density. The particles are initially distributed uniformly throughout the transverse $x-y$ plane. For this simulation, we tracked $1.2 \times 10^{7}$ molecules with $L^{2} / \sigma^{2}=10^{-5}$. The density is estimated on a grid of $400 \times 400$ bins.

a full exploration of the complex dynamics, but did not correspond to the actual parameter regime.

Against this background, as a next step to further examine our alignment ansatz, we now take into account that the force responsible for the molecular vibrations is much larger than all the other forces, and, consequently, the timescale of these vibrations far shorter than all other timescales. Hence, we may take the distance between a molecule's atoms to be frozen and consider a rigid model of the molecule. Simulation using a rigid model are numerically much easier to carry out. For the situation in question, where the electric and magnetic fields are all transverse, the motion of one molecule is described by three dynamical variables, namely the normalized transverse center-of-mass coordinates $x_{n}$ and $y_{n}$, and the angle of the dipole moment with respect to the relevant field $\theta$, and their conjugated momenta.

In simulations using the rigid molecule model, the initial centroid velocity spread of the molecules is chosen in accordance with the thermal equilibrium temperature, and so is the initial rotational velocity spread of the dipolemoment angle, $d \theta / d \tau$, while the starting angle $\theta$ itself is taken to be initially aligned with $\theta_{E}$ (for the case of an electric dipole moment). Simulations result for the rigidmolecule model with the parameters of Fig. 18 (center) are shown in Fig. 26, at a time corresponding to $n_{\text {osc. }}=1$. Both figures, obtained with the aligned dipole-moment model and with the rigid-molecule model, respectively, exhibit a similar annular structure in phase space, with a density maximum located at the same radial position. This similarity of results lends further support to our simplifying alignment ansatz.

In this Appendix, we have shown that the molecular electric or magnetic dipole moments $\vec{p}$ or $\vec{\mu}$ tend to align with the corresponding external field, as the external field is increased. In particular, we have demonstrated the validity of the alignment assumption in various simulations performed under different assumptions (e.g., with and without internal molecule vibrations) and timescales (mean field approximation or resolving individual bunch passages).

However, an exact simulation of repeated passages of short bunches through a region populated by EDM or MDM molecules with internal vibration modes requires the development of additional methods and tools to investigate the complete nonlinear dynamics of this complicated multiscale system.

[1] J. Wenninger for the Operations group, LHC Report: Operation with Holes, CERN News.

[2] B. Salvant, 16L2. BE-ABP information meeting, 2017.

[3] B. Salvant, S. Antipov, G. Arduini, N. Biancacci, X. Buffat, L. Carver, P. Collier, A. Gorzawski, W. Hfle, G. Iadarola, G. Kotzian, A. Lechner, T. Levens, L. Mether, D. Mirarchi, E. Mtral, G. Rumolo, and D. Valuch, Experimental characterization of a fast instability linked to losses in the 16L2 cryogenic half-cell in the CERN LHC, Report No. CERN-ACC-2018-175, THPAF058, 2018.

[4] F. Bordry, Summary of LHC performance workshop, Chamonix, 2018.

[5] J. M. Jimenez et al., Observations, analysis and mitigation of recurrent LHC beam dumps caused by fast losses in arc half-cell 16L2, Report No. CERN-ACC-2018-164, MOPMF053, 2018.

[6] Encyclopadia Britannica (Addison-Wesley, Reading, Massachusetts, 1993).

[7] D. Halliday, R. Resnick, and J. Walker, Fundamentals of Physics, 10th ed. (Wiley, New York, 2004).

[8] D. J. Griffiths, Introduction to Electrodynamics, 3rd ed. (Prentice-Hall, Upper Saddle River, NJ, 1999).

[9] M. Getzlaff, Fundamentals of Magnetism (SpringerVerlag, Berlin, 2008).

[10] W. S. C. Williams, Nuclear and Particles Physics (Oxford University Press, New York, 1991).

[11] Wolfram Mathematica, https://www.wolfram.com/ mathematica.

[12] I. M. Kapchinskij and V. V. Vladimirskij, Limitations of proton beam current in a strong focusing linear accelerator associated with the beam space charge, in the 2nd International Conference on High-Energy Accelerators (CERN, Geneva, 1959), pp. 274-287.

[13] I. Hofmann, L. J. Laslett, L. Smith, and I. Haber, Stability of the Kapchinskij-Vladimirskij (K-V) distribution in long periodic transport systems, Part. Accel. 13, 145 (1982), http://cds.cern.ch/record/140869/files/p145.pdf.

[14] E. Benedetto and F. Zimmermann, Dynamics of the electron pinch and incoherent tune shift induced by electron cloud, in Proceedings of the 9th European 
Particle Accelerator Conference, Lucerne, 2004 (EPS-AG, Lucerne, 2004), p. 7.

[15] E. Benedetto, G. Franchetti, and F. Zimmermann, Incoherent Effects of Electron Clouds in Proton Storage Rings, Phys. Rev. Lett. 97, 034801 (2006).

[16] F. Zimmermann and G. Franchetti, Electron-cloud pinch dynamics in presence of lattice magnet fields, Conf. Proc. C 110904, 586 (2011), https://accelconf.web.cern.ch/ IPAC2011/papers/MOPS001.pdf.

[17] F. Family and D. P. Landau, Kinetics of Aggregation and Gelation (North-Holland, Amsterdam, 2012).

[18] R. Jullien and R. Botet, Aggregation and Fractal Aggregates (World Scientific, Singapore, 1987).

[19] P. M. Mors, R. Botet and R. Jullien, Cluster-cluster aggregation with dipolar interactions, J. Phys. A 20, L975 (1987).

[20] R. Pastor-Satorras and J. M. Rubí, Particle-cluster aggregation with dipolar interactions, Phys. Rev. E 51, 5994 (1995).

[21] M.-Carmen Miguel and R. Pastor-Satorras, Kinetic growth of field-oriented chains in dipolar colloidal solutions, Phys. Rev. E 59, 826 (1999).

[22] J. D. Perry, L. S. Matthews and T. W. Hyde, Dipole-dipole interactions of charged-magnetic grains, IEEE Trans. Plasma Sci. 38, 792 (2010).

[23] Y. Baconnier, A. Poncet, and P. Tavares, Neutralization of accelerator beams by ionization of the residual gas, in
CERN Accelerator School: Course on General Accelerator Physics (CERN, Geneva, 1994), pp. 525-564.

[24] Y. Baconnier and G. Brianti, The stability of ions in bunched beam machines, in 1983 CERN 1st Accelerator School: Antiprotons for Colliding Beam Facilities (CERN, Geneva, 1980), p. 369.

[25] F. Zimmermann, J. T. Seeman, M. S. Zolotorev, and W. Stoeffl, Trapped macroparticles in electron storage rings, Report No. SLAC-95-6788, 1995.

[26] L. Mether, G. Iadarola, K. Poland, G. Rumolo, and G. Skripka, Multi-species electron-ion simulations and their application to the LHC, Report No. CERN-ACC-2019113, 2019, WEPTS050.

[27] D. G. Koshkarev and P. R. Zenkevich, Resonance of coupled transverse oscillations in two circular beams, Part. Accel. 3, 1 (1972), http://cds.cern.ch/record/1107898/files/ p1.pdf.

[28] T. O. Raubenheimer and F. Zimmermann, A fast beam-ion instability in linear accelerators and storage rings, Phys. Rev. E 52, 5487 (1995).

[29] G. V. Stupakov, T. O. Raubenheimer, and F. Zimmermann, Fast beam ion instability. 2. Effect of ion decoherence, Phys. Rev. E 52, 5499 (1995).

[30] R. A. Bosch, Spread-frequency model of the fast beam-ion instability in an electron storage ring, Phys. Rev. ST Accel. Beams 3, 034402 (2000). 